\title{
An Efficient Explicit Decoupled Group Method for Solving Two- Dimensional Fractional Burgers' Equation and Its Convergence Analysis
}

\author{
N. Abdi, ${ }^{1}$ H. Aminikhah $\left(\mathbb{D},{ }^{1,2}\right.$ A. H. Refahi Sheikhani $\mathbb{D}^{3}{ }^{3}$ J. Alavi, ${ }^{1}$ and M. Taghipour ${ }^{1}$ \\ ${ }^{1}$ Department of Applied Mathematics and Computer Science, Faculty of Mathematical Sciences, University of Guilan, P.O. Box 1914, \\ Rasht 41938, Iran \\ ${ }^{2}$ Center of Excellence for Mathematical Modelling, Optimization and Combinational Computing (MMOCC), University of Guilan, \\ P.O. Box 1914, Rasht 41938, Iran \\ ${ }^{3}$ Department of Applied Mathematics, Faculty of Mathematical Sciences, Lahijan Branch, Islamic Azad University, Lahijan, Iran
}

Correspondence should be addressed to H. Aminikhah; aminikhah@guilan.ac.ir

Received 17 November 2020; Revised 10 December 2020; Accepted 22 February 2021; Published 16 March 2021

Academic Editor: Manuel De León

Copyright (c) 2021 N. Abdi et al. This is an open access article distributed under the Creative Commons Attribution License, which permits unrestricted use, distribution, and reproduction in any medium, provided the original work is properly cited.

\begin{abstract}
In this paper, the Crank-Nicolson (CN) and rotated four-point fractional explicit decoupled group (EDG) methods are introduced to solve the two-dimensional time-fractional Burgers' equation. The EDG method is derived by the Taylor expansion and $45^{\circ}$ rotation of the Crank-Nicolson method around the $x$ and $y$ axes. The local truncation error of CN and EDG is presented. Also, the stability and convergence of the proposed methods are proved. Some numerical experiments are performed to show the efficiency of the presented methods in terms of accuracy and CPU time.
\end{abstract}

\section{Introduction}

Fractional calculus is a generalization of the integration and derivation of integer order operators to fractional order that allows us to describe a real system more accurately than integers. Although the fractional order of a real system may be low, it is yet considered as a fractional system. An important feature of fractional calculus is its nonlocality. The fractional derivative (and integrals) of a function is given by a definite integral, thus, it depends on the value of the function over the entire interval [1]. Researchers confirm the existence of interesting phenomena in nature, which cannot be modeled by classical differential equations. To cope with this problem, the nonlocality property of fractional derivative could be a beneficial tool to study our considered system. Fractional calculus has recently been used in various scientific and engineering fields [2-4]. Some fractional calculus applications in modeling and design of control systems are introduced in [5]. The most recent developments and trends in the use of fractional calculus in biomedicine and biology are presented by Ionescu et al. [6]. Based on the fractional calculate, Tang et al. [7] proposed a new four-element creep model; this model accords well with the experimental data of Changshan rock salt. Fractional calculus has an extraordinary potential in signal denoising [8]. Gong et al. discussed the generation conditions of chaotic behavior and proposed the adaptive synchronization control method for a class of fractionalorder financial system [9]. Numerous definitions of fractional derivative have been introduced in the literature, amongst are Riemann-Liouville, Caputo, and Caputo-Fabrizio [10]. The Caputo-Fabrizio fractional derivative on the contrary of other derivatives has a nonsingular kernel. Hence, it has been considered by many researchers [11-15]. Since to obtain the exact solution of fractional differential equations is very difficult and sometimes impossible, it is usually approximated by a numerical method such as finite difference method [16-18], finite volume methods [19], and spectral method [20]. 
Burgers' equation as a nonlinear partial differential equation is widely used in various areas such as fluid mechanics, gas dynamics, and traffic flow which combine nonlinear propagation effects with diffusive one.

In this paper, we consider the following two dimensional time-fractional Burgers' equation:

$$
\begin{gathered}
\frac{\partial^{\alpha} u}{\partial t^{\alpha}}+u\left(\frac{\partial u}{\partial x}+\frac{\partial u}{\partial y}\right)= \\
v\left(\frac{\partial^{2} u}{\partial x^{2}}+\frac{\partial^{2} u}{\partial y^{2}}\right)+f(x, y, t), \\
(x, y) \in \Omega, 0<t<T,
\end{gathered}
$$

with the initial condition

$$
u(x, y, 0)=u_{0}(x, y),(x, y) \in \Omega,
$$

and the boundary condition

$$
u(x, y, t)=0,(x, y) \in \partial \Omega, 0 \leq t \leq T
$$

where $v=1 / \operatorname{Re}, \operatorname{Re}$ is Reynolds number characterizing the strength of viscosity, $\Omega=(0,1) \times(0,1), 0<\alpha<1$, and the term $\partial^{\alpha} u / \partial t^{\alpha}$ denotes the $\alpha$ order Caputo-Fabrizio fractional derivative of the function $u(x, y, t)$ defined as:

$$
\frac{\partial^{\alpha} u(x, y, t)}{\partial t^{\alpha}}=\frac{M(\alpha)}{1-\alpha} \int_{0}^{t} \frac{\partial u(x, y, s)}{\partial s} e^{-\frac{\alpha}{1-\alpha}(t-s)} d s,
$$

where $M(\alpha)$ is a normalization function such that $M(0)$ $=M(1)=1$.

We apply the finite difference method to solve Equations (1)-(3). In finite difference, to find the value corresponding to each grid point, it is used natural ordering (indexing the grid of point from left to right and bottom to top by point to point) or group to group. There are several different methods to order the interior mesh point such as natural ordering, diagonal ordering, and alternating diagonal ordering [21]. Different linear systems would be produced by different arrangements of the grid points.

We propose two finite difference schemes. The first scheme is given by the Crank-Nicolson difference method (by natural ordering). In this scheme, to obtain a more accurate numerical solution, we should use a smaller mesh size, which requires more storage space and computing time. In order to fix this problem and accelerate the convergence, we use the explicit decoupled group (EDG) method introduced by Abdullah in 1990 [22]. Many studies have been done in reference to the EDG method for examples [2326]. The EDG method is based on rotating the Crank-Nicolson difference scheme and group ordering of grid points. Applying the EDG method to the Crank-Nicolson difference scheme result in a new scheme in which the dimension of the system is half of the dimension of the system generated by the Crank-Nicolson difference scheme. On this account, half of the grid points are obtained and the rest can be calculated directly. Consequently, the EDG method can be favourably used to reduce the computational cost. In addition, it is worth to notice that we can take advantage of parallel computers to run it.

The rest of the paper is organized as follows. In Section 2, the Crank-Nicolson difference scheme will be applied to Equations (1)-(3), and also, we give the truncation error. In Section 3, we describe the formulation of the EDG method. The stability of these schemes is discussed in Section 4. In Section 5, we analyze the convergence of these schemes. Numerical examples are carried out to verify the high efficiency of our method in Section 6. Finally, the paper ends with a brief conclusion in Section 7 .

\section{U'The Crank-Nicolson Difference Scheme}

For the numerical solution of Equations (1)-(3), we introduce a uniform grid of mesh points $\left(x_{i}, y_{j}, t_{n}\right)$ with $x_{i}=i \Delta x$, $i=0,1, \cdots, I, y_{j}=j \Delta y, j=0,1, \cdots, J$, and $t_{n}=n \Delta t, n=0,1$, $\cdots, N$.

Using the Crank-Nicolson approximation to Equations (1)-(3), we have

$$
\begin{aligned}
\left(\frac{\partial^{\alpha} u}{\partial t^{\alpha}}\right)_{i, j}^{n+1 / 2} & +\frac{\left(u u_{x}\right)_{i, j}^{n+1}+\left(u u_{x}\right)_{i, j}^{n}}{2}+\frac{\left(u u_{y}\right)_{i, j}^{n+1}+\left(u u_{y}\right)_{i, j}^{n}}{2} \\
= & v\left(\frac{\left(u_{x x}\right)_{i, j}^{n+1}+\left(u_{x x}\right)_{i, j}^{n}}{2}+\frac{\left(u_{y y}\right)_{i, j}^{n+1}+\left(u_{y y}\right)_{i, j}^{n}}{2}\right) \\
& +f_{i, j}^{n+1 / 2} .
\end{aligned}
$$

We use the following linearization technique for nonlinear term $\left(u u_{x}\right)^{n+1}$ and $\left(u u_{y}\right)^{n+1}$ [27]

$$
\begin{aligned}
& \left(u u_{x}\right)^{n+1} \approx u^{n+1} u_{x}^{n}+u^{n} u_{x}^{n+1}-u^{n} u_{x}^{n}, \\
& \left(u u_{y}\right)^{n+1} \approx u^{n+1} u_{y}^{n}+u^{n} u_{y}^{n+1}-u^{n} u_{y}^{n} .
\end{aligned}
$$

Substituting the above approximation into Equation (5), we yield

$$
\begin{aligned}
\left(\frac{\partial^{\alpha} u}{\partial t^{\alpha}}\right)_{i, j}^{n+1 / 2}+ & \frac{u_{i, j}^{n+1}\left(u_{x}\right)_{i, j}^{n}+u_{i, j}^{n}\left(u_{x}\right)_{i, j}^{n+1}}{2}+\frac{u_{i, j}^{n+1}\left(u_{y}\right)_{i, j}^{n}+u_{i, j}^{n}\left(u_{y}\right)_{i, j}^{n+1}}{2} \\
= & v\left(\frac{\left(u_{x x}\right)_{i, j}^{n+1}+\left(u_{x x}\right)_{i, j}^{n}}{2}+\frac{\left(u_{y y}\right)_{i, j}^{n+1}+\left(u_{y y}\right)_{i, j}^{n}}{2}\right) \\
& +f_{i, j}^{n+1 / 2} .
\end{aligned}
$$

A discrete approximation to the ${ }_{0}^{C F} D_{t}^{\alpha} u(x, y, t)$ at $\left(x_{i}, y_{j}\right.$, $\left.t_{n+1 / 2}\right)$ can be obtained by the following approximation 


$$
\begin{aligned}
& \frac{\partial^{\alpha} u\left(x_{i}, y_{j}, t_{n+1 / 2}\right)}{\partial t^{\alpha}}=\frac{1}{1-\alpha} \int_{0}^{t_{n+\frac{1}{2}}} \frac{\partial u\left(x_{i}, y_{j}, s\right)}{\partial s} e^{-\frac{\alpha}{1-\alpha}\left(t_{n+\frac{1}{2}}-s\right)} d s \\
& \left.=\frac{1}{1-\alpha}\left[\int_{0}^{t_{n}} \frac{\partial u\left(x_{i}, y_{j}, s\right)}{\partial s} e^{-\frac{\alpha}{1-\alpha}\left(t_{n+\frac{1}{2}}-s\right.}\right) d s+\int_{t_{n}}^{t_{n+\frac{1}{2}}} \frac{\partial u\left(x_{i}, y_{j}, s\right)}{\partial s} e^{-\frac{\alpha}{1-\alpha}\left(t_{n+\frac{1}{2}}-s\right)} d s\right] \\
& =\frac{1}{1-\alpha}\left[\sum_{k=1}^{n} \int_{t_{k-1}}^{t_{k}}\left(\frac{u_{i, j}^{k}-u_{i, j}^{k-1}}{\Delta t}+\left(s-t_{k-\frac{1}{2}}\right) u_{t t}\left(x_{i}, y_{j}, c_{k}\right)\right) e^{-\frac{\alpha}{1-\alpha}\left(t_{n+\frac{1}{2}}-s\right)} d s\right. \\
& \left.+\int_{t_{n}}^{t_{n+\frac{1}{2}}}\left(\frac{u_{i, j}^{n+1 / 2}-u_{i, j}^{n}}{\Delta t / 2}+O(\Delta t)\right) e^{-\frac{\alpha}{1-\alpha}\left(t_{n+\frac{1}{2}} \frac{-s}{}\right)} d s\right] \\
& =\frac{1}{1-\alpha}\left[\sum_{k=1}^{n} \int_{t_{k-1}}^{t_{k}}\left(\frac{u_{i, j}^{k}-u_{i, j}^{k-1}}{\Delta t}+\left(s-t_{k-\frac{1}{2}}\right) u_{t t}\left(x_{i}, y_{j}, c_{k}\right)\right) e^{-\frac{\alpha}{1-\alpha}\left(t_{n+\frac{1}{2}}-s\right)} d s\right. \\
& \left.\quad+\int_{t_{n}}^{t_{n+\frac{1}{2}}}\left(\frac{u_{i, j}^{n+1}+u_{i, j}^{n} / 2-u_{i, j}^{n}}{\Delta t / 2}+O(\Delta t)\right) e^{-\frac{\alpha}{1-\alpha}\left(t_{n+\frac{1}{2}-s}\right)} d s\right],
\end{aligned}
$$

where $c_{k} \in\left(t_{k-1}, t_{k}\right)$. Then,

$$
\begin{aligned}
& \frac{\partial^{\alpha} u\left(x_{i}, y_{j}, t_{n+1 / 2}\right)}{\partial t^{\alpha}}=\frac{1}{1-\alpha} \sum_{k=1}^{n} \int_{t_{k-1}}^{t_{k}} \frac{u_{i, j}^{k}-u_{i, j}^{k-1}}{\Delta t} e^{-\frac{\alpha}{1-\alpha}\left(t_{n+\frac{1}{2}}-s\right)} d s \\
& +\frac{1}{1-\alpha} \sum_{k=1}^{n} \int_{t_{k-1}}^{t_{k}}\left(s-t_{k-\frac{1}{2}}\right) u_{t t}\left(x_{i}, y_{j}, c_{k}\right) e^{-\frac{\alpha}{1-\alpha}\left(t_{n+\frac{1}{2}}-s\right)} d s \\
& +\frac{1}{1-\alpha} \int_{t_{n}}^{t_{n+\frac{1}{2}}}\left(\frac{u_{i, j}^{n+1}-u_{i, j}^{n}}{\Delta t}+O(\Delta t)\right) e^{-\frac{\alpha}{1-\alpha}\left(t_{n+\frac{1}{2}}-s\right)} d s \\
& =\frac{1}{\alpha \Delta t}\left\{\left(u_{i, j}^{n+1}-u_{i, j}^{n}\right)\left(1-e^{-\frac{\alpha}{1-\alpha}\left(\frac{1}{2}\right) \Delta t}\right)+\sum_{k=1}^{n}\left[u_{i, j}^{k}-u_{i, j}^{k-1}\right]\right. \\
& \left.\cdot\left[e^{-\frac{\alpha}{1-\alpha}\left(n-k+\frac{1}{2}\right) \Delta t}-e^{-\frac{\alpha}{1-\alpha}\left(n-k+\frac{3}{2}\right) \Delta t}\right]\right\}+R_{1}+R_{2},
\end{aligned}
$$

which

$R_{1}=\frac{1}{1-\alpha} \sum_{k=1}^{n} \int_{t_{k-1}}^{t_{k}}\left(s-t_{k-\frac{1}{2}}\right) u_{t t}\left(x_{i}, y_{j}, c_{k}\right) e^{-\frac{\alpha}{1-\alpha}\left(t_{n+\frac{1}{2}}-s\right)} d s$

and

$$
R_{2}=\frac{1}{\alpha}\left(1-e^{-\frac{\alpha}{1-\alpha}\left(\frac{1}{2}\right) \Delta t}\right) O(\Delta t)
$$

$$
\begin{aligned}
& \leq \frac{\max _{1 \leq k \leq n}\left|u_{t t}\left(x_{i}, y_{j}, c_{k}\right)\right| \Delta t}{2(1-\alpha)} \sum_{k=1}^{n} \int_{t_{k-1}}^{t_{k}} e^{-\frac{\alpha}{1-\alpha}\left(t_{n+\frac{1}{2}}-s\right)} d s \\
= & \frac{\max _{1 \leq k \leq n}\left|u_{t t}\left(x_{i}, y_{j}, c_{k}\right)\right| \Delta t}{2 \alpha} \sum_{k=1}^{n}\left(e^{-\frac{\alpha}{1-\alpha}\left(n-k+\frac{1}{2}\right) \Delta t}-e^{-\frac{\alpha}{1-\alpha}\left(n-k+\frac{3}{2}\right) \Delta t}\right) \\
= & \frac{\max _{1 \leq k \leq n}\left|u_{t t}\left(x_{i}, y_{j}, c_{k}\right)\right| \Delta t}{2 \alpha}\left(e^{-\frac{\alpha}{1-\alpha}\left(\frac{1}{2}\right) \Delta t}-e^{-\frac{\alpha}{1-\alpha}\left(n+\frac{1}{2}\right) \Delta t}\right) \\
\approx & \frac{\max _{1 \leq k \leq n}\left|u_{t t}\left(x_{i}, y_{j}, c_{k}\right)\right| \Delta t}{2 \alpha}\left(\frac{\alpha}{1-\alpha}\left(n+\frac{1}{2}-\frac{1}{2}\right) \Delta t\right) \\
= & \frac{\max _{1 \leq k \leq n}\left|u_{t t}\left(x_{i}, y_{j}, c_{k}\right)\right| \Delta t}{2(1-\alpha)}=O \Delta t \\
= & \frac{1 \leq k \leq n}{2(1-\alpha)}\left|u_{t t}\left(x_{i}, y_{j}, c_{k}\right)\right| T \Delta t
\end{aligned}
$$

By setting

$$
w_{k}=e^{-\frac{\alpha}{1-\alpha}\left(k-\frac{1}{2}\right) \Delta t}-e^{-\frac{\alpha}{1-\alpha}\left(k+\frac{1}{2}\right) \Delta t},
$$

finally, we obtain

$$
\begin{aligned}
\frac{\partial^{\alpha} u\left(x_{i}, y_{j}, t_{n+1 / 2}\right)}{\partial t^{\alpha}}= & \frac{1}{\alpha \Delta t}\left[-w_{n} u_{i, j}^{0}+\sum_{k=1}^{n-1}\left(w_{n-k+1}-w_{n-k}\right) u_{i, j}^{k}\right. \\
& \left.+w_{1} u_{i, j}^{n}+\left(u_{i, j}^{n+1}-u_{i, j}^{n}\right)\left(1-e^{-\frac{\alpha}{1-\alpha}\left(\frac{1}{2}\right) \Delta t}\right)\right] \\
& +O(\Delta t) .
\end{aligned}
$$

Besides, utilizing the Taylor expansion, we have

$$
\begin{aligned}
\frac{\partial^{2} u\left(x_{i}, y_{j}, t_{n+1 / 2}\right)}{\partial x^{2}}= & \frac{1}{2}\left[\frac{u_{i+1, j}^{n+1}-2 u_{i, j}^{n+1}+u_{i-1, j}^{n+1}}{(\Delta x)^{2}}+\frac{u_{i+1, j}^{n}-2 u_{i, j}^{n}+u_{i-1, j}^{n}}{(\Delta x)^{2}}\right] \\
& +O\left(\Delta t^{2}+\Delta x^{2}\right),
\end{aligned}
$$

$$
\begin{aligned}
\frac{\partial^{2} u\left(x_{i}, y_{j}, t_{n+1 / 2}\right)}{\partial y^{2}}= & \frac{1}{2}\left[\frac{u_{i, j+1}^{n+1}-2 u_{i, j}^{n+1}+u_{i, j-1}^{n+1}}{(\Delta y)^{2}}+\frac{u_{i, j+1}^{n}-2 u_{i, j}^{n}+u_{i, j-1}^{n}}{(\Delta y)^{2}}\right] \\
& +O\left(\Delta t^{2}+\Delta y^{2}\right),
\end{aligned}
$$




$$
\begin{aligned}
\begin{aligned}
u\left(x_{i}, y_{j}, t_{n+\frac{1}{2}}\right) & \frac{\partial u\left(x_{i}, y_{j}, t_{k+1 / 2}\right)}{\partial x} \\
= & \frac{1}{4 \Delta x}\left[u_{i, j}^{n}\left(u_{i+1, j}^{n+1}-u_{i-1, j}^{n+1}\right)+u_{i, j}^{n+1}\left(u_{i+1, j}^{n}-u_{i-1, j}^{n}\right)\right] \\
& +O\left(\Delta t^{2}+\Delta x^{2}\right),
\end{aligned} \\
\begin{aligned}
u\left(x_{i}, y_{j}, t_{n+\frac{1}{2}}\right) \frac{\partial u\left(x_{i}, y_{j}, t_{n+1 / 2}\right)}{\partial y} \\
=
\end{aligned} \\
\quad \frac{1}{4 \Delta y}\left[u_{i, j}^{n}\left(u_{i, j+1}^{n+1}-u_{i, j-1}^{n+1}\right)+u_{i, j}^{n+1}\left(u_{i, j+1}^{n}-u_{i, j-1}^{n}\right)\right] \\
+O\left(\Delta t^{2}+\Delta y^{2}\right) .
\end{aligned}
$$

Using the Equations (14)-(18), we derive the following difference scheme which is accurate of the order $O\left(\Delta t+\Delta x^{2}\right.$ $\left.+\Delta y^{2}\right)$

$$
\begin{aligned}
\frac{1}{\alpha \Delta t}[- & \left.w_{n} U_{i, j}^{0}+\sum_{k=1}^{n-1}\left(w_{n-k+1}-w_{n-k}\right) U_{i, j}^{k}+w_{1} U_{i, j}^{n}+\left(U_{i, j}^{n+1}-U_{i, j}^{n}\right) D_{0}\right] \\
= & \frac{v}{2}\left[\frac{U_{i+1, j}^{n+1}-2 U_{i, j}^{n+1}+U_{i-1, j}^{n+1}}{(\Delta x)^{2}}+\frac{\left.U_{i+1, j}^{n}-2 U_{i, j}^{n}+U_{i-1, j}^{n}\right]}{(\Delta x)^{2}}\right] \\
& +\frac{v}{2}\left[\frac{U_{i, j+1}^{n+1}-2 U_{i, j}^{n+1}+U_{i, j-1}^{n+1}}{(\Delta y)^{2}}+\frac{U_{i, j+1}^{n}-2 U_{i, j}^{n}+U_{i, j-1}^{n}}{(\Delta y)^{2}}\right] \\
& -\frac{1}{4 \Delta x}\left[U_{i, j}^{n}\left(U_{i+1, j}^{n+1}-U_{i-1, j}^{n+1}\right)+U_{i, j}^{n+1}\left(U_{i+1, j}^{n}-U_{i-1, j}^{n}\right)\right] \\
& -\frac{1}{4 \Delta y}\left[U_{i, j}^{n}\left(U_{i, j+1}^{n+1}-U_{i, j-1}^{n+1}\right)+U_{i, j}^{n+1}\left(U_{i, j+1}^{n}-U_{i, j-1}^{n}\right)\right] \\
& +f\left(x_{i}, y_{j}, t_{n+\frac{1}{2}}\right),
\end{aligned}
$$

where $D_{0}=\left(1-e^{-(\alpha / 1-\alpha)(1 / 2) \Delta t}\right)$ and $U_{i, j}^{n}$ represents the approximate solution of Equation (1).

After simplification, we obtain

$$
\begin{aligned}
& \left(D_{0}+\frac{\alpha \Delta t}{4 \Delta x}\left(U_{i+1, j}^{n}-U_{i-1, j}^{n}\right)+\frac{\alpha \Delta t}{4 \Delta y}\left(U_{i, j+1}^{n}-U_{i, j-1}^{n}\right)+\frac{\alpha \Delta t v}{(\Delta x)^{2}}+\frac{\alpha \Delta t v}{(\Delta y)^{2}}\right) U_{i, j}^{n+1} \\
& -\left(\frac{\alpha \Delta t v}{2(\Delta x)^{2}}-U_{i, j}^{n} \frac{\alpha \Delta t}{4 \Delta x}\right) U_{i+1, j}^{n+1}-\left(\frac{\alpha \Delta t v}{2(\Delta x)^{2}}+U_{i, j}^{n} \frac{\alpha \Delta t}{4 \Delta x}\right) U_{i-1, j}^{n+1} \\
& \quad-\left(\frac{\alpha \Delta t v}{2(\Delta y)^{2}}-U_{i, j}^{n} \frac{\alpha \Delta t}{4 \Delta y}\right) U_{i, j+1}^{n+1}-\left(\frac{\alpha \Delta t v}{2(\Delta y)^{2}}+U_{i, j}^{n} \frac{\alpha \Delta t}{4 \Delta y}\right) U_{i, j-1}^{n+1} \\
& =\left(D_{0}-\frac{\alpha \Delta t v}{(\Delta x)^{2}}-\frac{\alpha \Delta t v}{(\Delta y)^{2}}-w_{1}\right) U_{i, j}^{n}+\frac{\alpha \Delta t v}{2(\Delta x)^{2}} U_{i+1, j}^{n}+\frac{\alpha \Delta t v}{2(\Delta x)^{2}} U_{i-1, j}^{n} \\
& \quad+\frac{\alpha \Delta t v}{2(\Delta y)^{2}} U_{i, j+1}^{n}+\frac{\alpha \Delta t v}{2(\Delta y)^{2}} U_{i, j-1}^{n}+w_{n} U_{i, j}^{0}-\sum_{k=1}^{n-1}\left(w_{n-k+1}-w_{n-k}\right) U_{i, j}^{k}+(\alpha \Delta t) f_{i, j}^{n+\frac{1}{2}}
\end{aligned}
$$

\section{Fractional Explicit Decoupled Group Method}

Another approximate formula for Equation (1) is obtained by Taylor's expansion and rotating Equation (20), $45^{\circ}$ degrees clockwise around the $x-y$ axis. The Crank-Nicolson rotation formula for Equation (1) is as follows

$$
\begin{aligned}
\frac{1}{\alpha \Delta t}[ & \left.-w_{n} U_{i, j}^{0}+\sum_{k=1}^{n-1}\left(w_{n-k+1}-w_{n-k}\right) U_{i, j}^{k}+w_{1} U_{i, j}^{n}+\left(U_{i, j}^{n+1}-U_{i, j}^{n}\right) D_{0}\right] \\
= & \frac{v}{4}\left[\frac{U_{i-1, j+1}^{n+1}-2 U_{i, j}^{n+1}+U_{i+1, j-1}^{n+1}}{h^{2}}+\frac{\left.U_{i-1, j+1}^{n}-2 U_{i, j}^{n}+U_{i+1, j-1}^{n}\right]}{h^{2}}\right] \\
& +\frac{v}{4}\left[\frac{U_{i+1, j+1}^{n+1}-2 U_{i, j}^{n+1}+U_{i-1, j-1}^{n+1}}{h^{2}}+\frac{U_{i+1, j+1}^{n}-2 U_{i, j}^{n}+U_{i-1, j-1}^{n}}{h^{2}}\right] \\
& -\frac{1}{4 h}\left[\frac{U_{i, j}^{n}}{2}\left(\left(U_{i+1, j+1}^{n+1}+U_{i+1, j-1}^{n+1}\right)-\left(U_{i-1, j-1}^{n+1}+U_{i-1, j+1}^{n+1}\right)\right)\right. \\
& \left.+\frac{U_{i, j}^{n+1}}{2}\left(\left(U_{i+1, j+1}^{n}+U_{i+1, j-1}^{n}\right)-\left(U_{i-1, j-1}^{n}+U_{i-1, j+1}^{n}\right)\right)\right] \\
& -\frac{1}{4 h}\left[\frac{U_{i, j}^{n}}{2}\left(\left(U_{i-1, j+1}^{n+1}+U_{i+1, j+1}^{n+1}\right)-\left(U_{i-1, j-1}^{n+1}+U_{i+1, j-1}^{n+1}\right)\right)\right. \\
& \left.+\frac{U_{i, j}^{n+1}}{2}\left(\left(U_{i-1, j+1}^{n}+U_{i+1, j+1}^{n}\right)-\left(U_{i-1, j-1}^{n}+U_{i+1, j-1}^{n}\right)\right)\right] \\
& +f\left(x_{i}, y_{j}, t_{n+\frac{1}{2}}\right),
\end{aligned}
$$

where $h=\Delta x=\Delta y$. Similarly, the above-rotated difference scheme is accurate of order $O\left(\Delta t+\Delta x^{2}+\Delta y^{2}\right)$. On simplification with $r_{x}=\alpha \Delta t / 4 h$ and $r_{x x}=\alpha \Delta t v / 2 h^{2}$, the following equation is obtained

$$
\begin{aligned}
( & \left.D_{0}+r_{x}\left(U_{i+1, j+1}^{n}-U_{i-1, j-1}^{n}\right)+2 r_{x x}\right) U_{i, j}^{n+1}-\frac{r_{x x}}{2} U_{i+1, j-1}^{n+1} \\
& -\frac{r_{x x}}{2} U_{i-1, j+1}^{n+1}-\left(\frac{r_{x x}}{2}-r_{x} U_{i, j}^{n}\right) U_{i+1, j+1}^{n+1}-\left(\frac{r_{x x}}{2}+r_{x} U_{i, j}^{n}\right) U_{i-1, j-1}^{n+1} \\
= & \left(D_{0}-2 r_{x x}-w_{1}\right) U_{i, j}^{n}+\frac{r_{x x}}{2} U_{i+1, j-1}^{n}+\frac{r_{x x}}{2} U_{i-1, j+1}^{n}+\frac{r_{x x}}{2} U_{i+1, j+1}^{n} \\
& +\frac{r_{x x}}{2} U_{i-1, j-1}^{n}+w_{n} U_{i, j}^{0}-\sum_{k=1}^{n-1}\left(w_{n-k+1}-w_{n-k}\right) U_{i, j}^{k}+(\alpha \Delta t) f_{i, j}^{n+1 / 2} .
\end{aligned}
$$

Utilizing Equation (22) to any group of four points on the solution domain gives a $(4 \times 4)$ system as follows

$$
\left[\begin{array}{cccc}
a_{i, j} & -c_{i, j} & 0 & 0 \\
-d_{i+1, j+1} & a_{i+1, j+1} & 0 & 0 \\
0 & 0 & a_{i+1, j} & -b \\
0 & 0 & -b & a_{i, j+1}
\end{array}\right]\left[\begin{array}{c}
U_{i, j}^{n+1} \\
U_{i+1, j+1}^{n+1} \\
U_{i+1, j}^{n+1} \\
U_{i, j+1}^{n+1}
\end{array}\right]=\left[\begin{array}{c}
r h s_{i, j} \\
r h s_{i+1, j+1} \\
r h s_{i+1, j} \\
r h s_{i, j+1}
\end{array}\right],
$$

where

$$
\begin{aligned}
a_{i, j} & =D_{0}+r_{x}\left(U_{i+1, j+1}^{n}-U_{i-1, j-1}^{n}\right)+2 r_{x x}, c_{i, j}=\frac{r_{x x}}{2}-r_{x} U_{i, j}^{n}, d_{i, j} \\
& =\frac{r_{x x}}{2}+r_{x} U_{i, j}^{n}, b=\frac{r_{x x}}{2},
\end{aligned}
$$




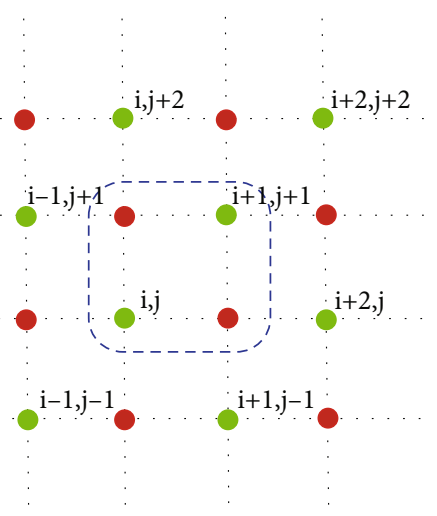

(a)

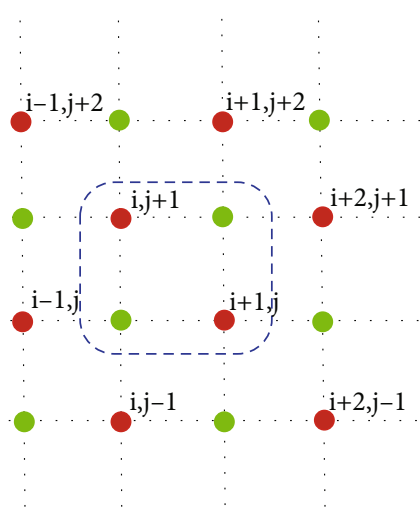

(b)

Figure 1: Grid point on $x-y$ plane. Computational molecule of Equation (27) (a) and computational molecule of Equation (28) (b).

with

$\left[\begin{array}{c}r h s_{i, j} \\ r h s_{i+1, j+1} \\ r h s_{i+1, j} \\ r h s_{i, j+1}\end{array}\right]=\left[\begin{array}{l}\left(b\left(U_{i+1, j-1}^{n+1}+U_{i-1, j+1}^{n+1}\right)+d_{i, j} U_{i-1, j-1}^{n+1}\right)+\left(D_{0}-2 r_{x x}-w_{1}\right) U_{i, j}^{n}+b\left(U_{i+1, j-1}^{n}+U_{i-1, j+1}^{n}+U_{i+1, j+1}^{n}+U_{i-1, j-1}^{n}\right)+\tau_{i, j} \\ \left(b\left(U_{i+2, j}^{n+1}+U_{i, j+2}^{n+1}\right)+c_{i+1, j+1} U_{i+2, j+2}^{n+1}\right)+\left(D_{0}-2 r_{x x}-w_{1}\right) U_{i+1, j+1}^{n}+b\left(U_{i+2, j}^{n}+U_{i, j+2}^{n}+U_{i+2, j+2}^{n}+U_{i, j}^{n}\right)+\tau_{i+1, j+1} \\ \left(b U_{i+2, j-1}^{n+1}+c_{i+1, j} U_{i+2, j+1}^{n+1}+d_{i+1, j} U_{i, j-1}^{n+1}\right)+\left(D_{0}-2 r_{x x}-w_{1}\right) U_{i+1, j}^{n}+b\left(U_{i+2, j-1}^{n}+U_{i, j+1}^{n}+U_{i+2, j+1}^{n}+U_{i, j-1}^{n}\right)+\tau_{i+1, j} \\ \left(b U_{i-1, j+2}^{n+1}+c_{i, j+1} U_{i+1, j+2}^{n+1}+d_{i, j+1} U_{i-1, j}^{n+1}\right)+\left(D_{0}-2 r_{x x}-w_{1}\right) U_{i+1, j}^{n}+b\left(U_{i+1, j}^{n}+U_{i-1, j+2}^{n}+U_{i+1, j+2}^{n}+U_{i-1, j}^{n}\right)+\tau_{i, j+1}\end{array}\right]$,

and

$$
\left[\begin{array}{c}
\tau_{i, j} \\
\tau_{i+1, j+1} \\
\tau_{i+1, j} \\
\tau_{i, j+1}
\end{array}\right]=\left[\begin{array}{c}
w_{n} U_{i, j}^{0}-\sum_{k=1}^{n-1}\left(w_{n-k+1}-w_{n-k}\right) U_{i, j}^{k}+(\alpha \Delta t) f_{i, j}^{n+\frac{1}{2}} \\
w_{n} U_{i+1, j+1}^{0}-\sum_{k=1}^{n-1}\left(w_{n-k+1}-w_{n-k}\right) U_{i+1, j+1}^{k}+(\alpha \Delta t) f_{i+1, j+1}^{n+\frac{1}{2}} \\
w_{n} U_{i+1, j}^{0}-\sum_{k=1}^{n-1}\left(w_{n-k+1}-w_{n-k}\right) U_{i+1, j}^{k}+(\alpha \Delta t) f_{i+1, j}^{n+\frac{1}{2}} \\
w_{n} U_{i, j+1}^{0}-\sum_{k=1}^{n-1}\left(w_{n-k+1}-w_{n-k}\right) U_{i, j+1}^{k}+(\alpha \Delta t) f_{i, j+1}^{n+\frac{1}{2}}
\end{array}\right]
$$

Equation (23) leads to a decoupled system of $2 \times 2$ equations in explicit form

$$
\left[\begin{array}{cc}
a_{i, j} & -c_{i, j} \\
-d_{i+1, j+1} & a_{i+1, j+1}
\end{array}\right]\left[\begin{array}{c}
U_{i, j}^{n+1} \\
U_{i+1, j+1}^{n+1}
\end{array}\right]=\left[\begin{array}{c}
r h s_{i, j} \\
r h s_{i+1, j+1}
\end{array}\right],
$$

and

$$
\left[\begin{array}{cc}
a_{i+1, j} & -b \\
-b & a_{i, j+1}
\end{array}\right]\left[\begin{array}{c}
U_{i+1, j}^{n+1} \\
U_{i, j+1}^{n+1}
\end{array}\right]=\left[\begin{array}{c}
r h s_{i+1, j} \\
r h s_{i, j+1}
\end{array}\right] .
$$

The computational molecule of Equations (27) and (28) is shown in Figure 1.

From Figure 1(a), it can be seen that Equation (27) is executed only by considering the green dots. On the contrary, Equation (28) only runs with red dots. Therefore, the implementation of these two equations is independent of each other, which makes the solution of Equation (1) consume less time.

In the EDG method, the grid points are divided into several groups. Each group consists of only two points of the grid (shown in Figure 2). We apply one of the Equation (27) or Equation (28) for each group in Figure 2. Therefore, half of the grid points (green dots) are calculated by the rotated finite-difference Equation (22). Before going to the next time level, we obtain other points of the grid (red dots) directly once by taking the Equation (20). 


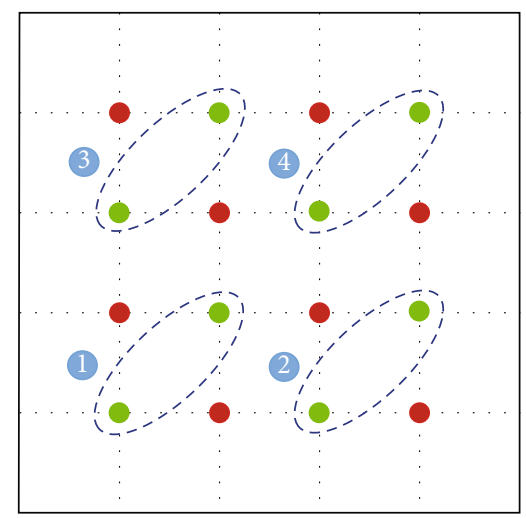

FIGURE 2: Group ordering for EDG method for $N=5$.

\section{Stability Analysis}

In this section, the stability of the finite difference method is investigated with the Von-Neumann analysis. We first give a lemma about $w_{j}$, which will be used in the stability analysis.

Lemma 1 (see [28]). The coefficients $w_{j}$ in Equation (13) satisfy the following properties.

$$
0 \leq w_{j} \leq C \Delta t
$$

and

$$
0 \leq w_{j}-w_{j+1} \leq C \Delta t w_{j}
$$

To investigate the stability of the difference scheme, the nonlinear term $u\left(u_{x}+u_{y}\right)$ in Equations (1)-(3) has been linearized by making the quantity $u$ to a local constant. Thus, the nonlinear term in Equation (1) converts into $\widehat{u}\left(u_{x}+u_{y}\right)$, and Equation (1) becomes:

$$
\frac{\partial^{\alpha} u}{\partial t^{\alpha}}+\widehat{u}\left(\frac{\partial u}{\partial x}+\frac{\partial u}{\partial y}\right)=v\left(\frac{\partial^{2} u}{\partial x^{2}}+\frac{\partial^{2} u}{\partial y^{2}}\right)+f(x, y, t)
$$

Let $\tilde{U}_{i, j}^{n}$ and $\bar{U}_{i, j}^{n}$ be the approximate solutions of Equations (20) and (22), respectively, and define

$$
\begin{gathered}
\rho_{i, j}^{n}=U_{i, j}^{n}-\tilde{U}_{i, j}^{n}, \\
\phi_{i, j}^{n}=U_{i, j}^{n}-\bar{U}_{i, j}^{n}, 1 \leq i \leq I, 1 \leq j \leq J, 1 \leq n \leq N .
\end{gathered}
$$

Then, by substituting Equation (32) into Equation (20), we have

$$
\begin{aligned}
& \left(\frac{v \alpha \Delta t}{(\Delta x)^{2}}+\frac{v \alpha \Delta t}{(\Delta y)^{2}}+D_{0}\right) \rho_{i, j}^{n+1}+\left(\frac{\alpha \Delta t \widehat{u}}{4 \Delta x}-\frac{v \alpha \Delta t}{2(\Delta x)^{2}}\right) \rho_{i+1, j}^{n+1} \\
& +\left(-\frac{\alpha \Delta t \widehat{u}}{4 \Delta x}-\frac{v \alpha \Delta t}{2(\Delta x)^{2}}\right) \rho_{i-1, j}^{n+1}+\left(\frac{\alpha \Delta t \widehat{u}}{4 \Delta y}-\frac{v \alpha \Delta t}{2(\Delta y)^{2}}\right) \rho_{i, j+1}^{n+1} \\
& +\left(-\frac{\alpha \Delta t \widehat{u}}{4 \Delta y}-\frac{v \alpha \Delta t}{2(\Delta y)^{2}}\right) \rho_{i, j-1}^{n+1}=\left(-\frac{v \alpha \Delta t}{(\Delta x)^{2}}-\frac{v \alpha \Delta t}{(\Delta y)^{2}}-w_{1}+D_{0}\right) \rho_{i, j}^{n} \\
& +\left(-\frac{\alpha \Delta t \widehat{u}}{4 \Delta x}+\frac{v \alpha \Delta t}{2(\Delta x)^{2}}\right) \rho_{i+1, j}^{n}+\left(\frac{\alpha \Delta t \widehat{u}}{4 \Delta x}+\frac{v \alpha \Delta t}{2(\Delta x)^{2}}\right) \rho_{i-1, j}^{n} \\
& +\left(-\frac{\alpha \Delta t \widehat{u}}{4 \Delta y}+\frac{v \alpha \Delta t}{2(\Delta y)^{2}}\right) \rho_{i, j+1}^{n}+\left(\frac{\alpha \Delta t \widehat{u}}{4 \Delta y}+\frac{v \alpha \Delta t}{2(\Delta y)^{2}}\right) \rho_{i, j-1}^{n}+w_{n} \rho_{i, j}^{0} \\
& \quad-\sum_{k=1}^{n-1}\left(w_{n-k+1}-w_{n-k}\right) \rho_{i, j}^{k} .
\end{aligned}
$$

Also by putting Equation (33) in Equation (22), we get

$$
\begin{aligned}
\left(D_{0}\right. & \left.+2 r_{x x}\right) \phi_{i, j}^{n+1}-\frac{r_{x x}}{2}\left(\phi_{i+1, j-1}^{n+1}-\phi_{i-1, j+1}^{n+1}\right)-\left(\frac{r_{x x}}{2}-\widehat{u} r_{x}\right) \phi_{i+1, j+1}^{n+1} \\
& -\left(\frac{r_{x x}}{2}+\widehat{u} r_{x}\right) \phi_{i-1, j-1}^{n+1}=\left(D_{0}-w_{1}+2 r_{x x}\right) \phi_{i, j}^{n} \\
& +\frac{r_{x x}}{2}\left(\phi_{i+1, j-1}^{n}+\phi_{i-1, j+1}^{n}\right)+\left(\frac{r_{x x}}{2}-\widehat{u} r_{x}\right) \phi_{i+1, j+1}^{n} \\
& +\left(\frac{r_{x x}}{2}+\widehat{u} r_{x}\right) \phi_{i-1, j-1}^{n}+w_{n} \phi_{i, j}^{0}-\sum_{k=1}^{n-1}\left(w_{n-k+1}-w_{n-k}\right) \phi_{i, j}^{k} .
\end{aligned}
$$

The Fourier series for $\rho^{n}(x, y)$ and $\phi^{n}(x, y)$ is

$$
\begin{aligned}
& \rho^{n}(x, y)=\sum_{m_{2}=-\infty}^{\infty} \sum_{m_{1}=-\infty}^{\infty} \xi_{n}\left(m_{1}, m_{2}\right) e^{i 2 \pi\left\{m_{1} x+m_{2} y\right\}}, \\
& \phi^{n}(x, y)=\sum_{m_{2}=-\infty}^{\infty} \sum_{m_{1}=-\infty}^{\infty} \eta_{n}\left(m_{1}, m_{2}\right) e^{i 2 \pi\left\{m_{1} x+m_{2} y\right\}},
\end{aligned}
$$

where $\iota=\sqrt{-1}$ and the amplication factors $\xi_{n}$ and $\eta_{n}$ are defined by

$$
\begin{gathered}
\xi_{n}\left(m_{1}, m_{2}\right)=\int_{0}^{1} \int_{0}^{1} e^{-\imath 2 \pi\left\{m_{1} \tau+m_{2} \varepsilon\right\}} \rho^{n}(\tau, \varepsilon) d \tau d \varepsilon, \\
\eta_{n}\left(m_{1}, m_{2}\right)=\int_{0}^{1} \int_{0}^{1} e^{-\imath 2 \pi\left\{m_{1} \tau+m_{2} \varepsilon\right\}} \phi^{n}(\tau, \varepsilon) d \tau d \varepsilon .
\end{gathered}
$$

Introducing the following norm

$$
\begin{aligned}
& \left\|\rho^{n}\right\|_{2}=\left(\sum_{j=1}^{J-1} \sum_{i=1}^{I-1} \Delta x \Delta y\left|\rho_{i, j}^{n}\right|^{2}\right)^{1 / 2}=\left(\int_{0}^{1} \int_{0}^{1}\left|\rho_{i, j}^{n}\right|^{2} d \tau d \varepsilon\right)^{1 / 2}, \\
& \left\|\phi^{n}\right\|_{2}=\left(\sum_{j=1}^{J-1} \sum_{i=1}^{I-1} \Delta x \Delta y\left|\phi_{i, j}^{n}\right|^{2}\right)^{1 / 2}=\left(\int_{0}^{1} \int_{0}^{1}\left|\phi_{i, j}^{n}\right|^{2} d \tau d \varepsilon\right)^{1 / 2} .
\end{aligned}
$$


By applying the Parseval's equality

$$
\begin{aligned}
& \int_{0}^{1} \int_{0}^{1}\left|\rho^{n}(\tau, \varepsilon)\right|^{2} d \tau d \varepsilon=\sum_{m_{2}=-\infty}^{\infty} \sum_{m_{1}=-\infty}^{\infty}\left|\xi_{n}\left(m_{1}, m_{2}\right)\right|^{2}, \\
& \int_{0}^{1} \int_{0}^{1}\left|\phi^{n}(\tau, \varepsilon)\right|^{2} d \tau d \varepsilon=\sum_{m_{2}=-\infty}^{\infty} \sum_{m_{1}=-\infty}^{\infty}\left|\eta_{n}\left(m_{1}, m_{2}\right)\right|^{2},
\end{aligned}
$$

we obtain

$$
\begin{aligned}
\left\|\rho^{n}\right\|_{2}^{2} & =\sum_{m_{2}=-\infty}^{\infty} \sum_{m_{1}=-\infty}^{\infty}\left|\xi_{n}\left(m_{1}, m_{2}\right)\right|^{2}, \\
\left\|\phi^{n}\right\|_{2}^{2} & =\sum_{m_{2}=-\infty}^{\infty} \sum_{m_{1}=-\infty}^{\infty}\left|\eta_{n}\left(m_{1}, m_{2}\right)\right|^{2} .
\end{aligned}
$$

According to the above analysis, we can suppose that the solution of Equations (34) and (35) has the following form

$$
\begin{aligned}
& \rho_{i, j}^{n}=\xi_{n} e^{l\left(\sigma_{x} i \Delta x+\sigma_{y} j \Delta y\right)}, \\
& \phi_{i, j}^{n}=\eta_{n} e^{l\left(\sigma_{x} i \Delta x+\sigma_{y} j \Delta y\right)},
\end{aligned}
$$

where $\sigma_{x}=2 m_{1} \pi, \sigma_{y}=2 m_{2} \pi$.

Lemma 2. If $w_{1} \leq D_{0}$ in Equation (20) and $\alpha \in(0,1)$, then we have

$$
\left|\xi_{n}\right| \leq\left|\xi_{0}\right|, n=1,2, \cdots, N
$$

where $\xi_{n}$ is defined in Equation (37).

Proof. Substituting $\rho_{i, j}^{n}=\xi_{n} e^{l\left(\sigma_{x} i \Delta x+\sigma_{y} j \Delta y\right)}$ into Equation (34), we have

$$
\begin{aligned}
& \left(\frac{v \alpha \Delta t}{(\Delta x)^{2}}+\frac{v \alpha \Delta t}{(\Delta y)^{2}}+D_{0}\right) \xi_{n+1} e^{t\left(\sigma_{x} i \Delta x+\sigma_{y} j \Delta y\right)} \\
& +\left(\frac{\alpha \Delta t \widehat{u}}{4 \Delta x}-\frac{v \alpha \Delta t}{2 \Delta x^{2}}\right) \xi_{n+1} e^{t\left(\sigma_{x}(i+1) \Delta x+\sigma_{y} j \Delta y\right)} \\
& +\left(-\frac{\alpha \Delta t \widehat{u}}{4 \Delta x}-\frac{v \alpha \Delta t}{2 \Delta x^{2}}\right) \xi_{n+1} e^{t\left(\sigma_{x}(i-1) \Delta x+\sigma_{y} j \Delta y\right)} \\
& +\left(\frac{\alpha \Delta t \widehat{u}}{4 \Delta y}-\frac{v \alpha \Delta t}{2 \Delta y^{2}}\right) \xi_{n+1} e^{t\left(\sigma_{x} i \Delta x+\sigma_{y}(j+1) \Delta y\right)} \\
& +\left(-\frac{\alpha \Delta t \widehat{u}}{4 \Delta y}-\frac{v \alpha \Delta t}{2 \Delta y^{2}}\right) \xi_{n+1} e^{t\left(\sigma_{x} i \Delta x+\sigma_{y}(j-1) \Delta y\right)} \\
& =\left(-\frac{v \alpha \Delta t}{(\Delta x)^{2}}-\frac{v \alpha \Delta t}{(\Delta y)^{2}}-w_{1}+D_{0}\right) \xi_{n} e^{l\left(\sigma_{x} i \Delta x+\sigma_{y} j \Delta y\right)} \\
& +\left(-\frac{\alpha \Delta t \widehat{u}}{4 \Delta x}+\frac{v \alpha \Delta t}{2(\Delta x)^{2}}\right) \xi_{n} e^{t\left(\sigma_{x}(i+1) \Delta x+\sigma_{y} j \Delta y\right)}
\end{aligned}
$$

$$
\begin{aligned}
& +\left(\frac{\alpha \Delta t \widehat{u}}{4 \Delta x}+\frac{v \alpha \Delta t}{2(\Delta x)^{2}}\right) \xi_{n} e^{l\left(\sigma_{x}(i-1) \Delta x+\sigma_{y} j \Delta y\right)} \\
& +\left(-\frac{\alpha \Delta t \widehat{u}}{4 \Delta y}+\frac{v \alpha \Delta t}{2(\Delta y)^{2}}\right) \xi_{n} e^{l\left(\sigma_{x} i \Delta x+\sigma_{y}(j+1) \Delta y\right)} \\
& +\left(\frac{\alpha \Delta t \widehat{u}}{4 \Delta y}+\frac{v \alpha \Delta t}{2(\Delta y)^{2}}\right) \xi_{n} e^{l\left(\sigma_{x} i \Delta x+\sigma_{y}(j-1) \Delta y\right)} \\
& +w_{n} \xi_{0} e^{l\left(\sigma_{x} i \Delta x+\sigma_{y} j \Delta y\right)} \\
& +\sum_{k=1}^{n-1}\left(w_{n-k+1}-w_{n-k}\right) \xi_{k} e^{l\left(\sigma_{x} i \Delta x+\sigma_{y} j \Delta y\right)},
\end{aligned}
$$

after simplifications, we can get

$$
\begin{aligned}
\xi_{n+1}[- & v \alpha \Delta t\left(-\frac{1}{(\Delta x)^{2}}-\frac{1}{(\Delta y)^{2}}+\frac{\cos \left(\sigma_{x} \Delta x\right)}{(\Delta x)^{2}}+\frac{\cos \left(\sigma_{y} \Delta y\right)}{(\Delta y)^{2}}\right) \\
& \left.+D_{0}+\iota \frac{\alpha \Delta t \hat{u}}{2}\left(\frac{\sin \left(\sigma_{x} \Delta x\right)}{\Delta x}+\frac{\sin \left(\sigma_{y} \Delta y\right)}{\Delta y}\right)\right] \\
= & \xi_{n}\left[\nu \alpha \Delta t\left(-\frac{1}{(\Delta x)^{2}}-\frac{1}{(\Delta y)^{2}}+\frac{\cos \left(\sigma_{x} \Delta x\right)}{(\Delta x)^{2}}+\frac{\cos \left(\sigma_{y} \Delta y\right)}{(\Delta y)^{2}}\right)\right. \\
& \left.+D_{0}-w_{1}-\iota \frac{\alpha \Delta t \hat{u}}{2}\left(\frac{\sin \left(\sigma_{x} \Delta x\right)}{\Delta x}+\frac{\sin \left(\sigma_{y} \Delta y\right)}{\Delta y}\right)\right] \\
& +w_{n} \xi_{0}-\sum_{k=1}^{n-1}\left(w_{n-k+1}-w_{n-k}\right) \xi_{k} .
\end{aligned}
$$

First, letting $n=0$ in Equation (45), we obtain

$$
\left|\xi_{1}\right|=|\gamma|\left|\xi_{0}\right|
$$

where

$$
\begin{aligned}
& \gamma=\frac{v \alpha \Delta t A+D_{0}-\iota(\alpha \Delta t \hat{u} / 2) B}{-v \alpha \Delta t A+D_{0}+\iota(\alpha \Delta t \hat{u} / 2) B}, \\
& A=-\frac{1}{(\Delta x)^{2}}-\frac{1}{(\Delta y)^{2}}+\frac{\cos \left(\sigma_{x} \Delta x\right)}{(\Delta x)^{2}}+\frac{\cos \left(\sigma_{y} \Delta y\right)}{(\Delta y)^{2}}, \\
& B=\frac{\sin \left(\sigma_{x} \Delta x\right)}{\Delta x}+\frac{\sin \left(\sigma_{y} \Delta y\right)}{\Delta y} .
\end{aligned}
$$

In the above expression, it is clear that the real part of the numerator is smaller than the real part of the denominator. Thus, the magnitude of the numerator is smaller than the denominator. So we have

$$
\left|\xi_{1}\right| \leq\left|\xi_{0}\right|
$$

Now, suppose that we have proved that $\left|\xi_{n}\right| \leq\left|\xi_{0}\right|, n=1$, $2, \cdots, m$. 
We should prove this for $n=m+1$. Using Equation (45), we get

$$
\begin{aligned}
& \left|\xi_{m+1}\right| \leq\left|\frac{v \alpha \Delta t A+D_{0}-w_{1}-\iota(\alpha \Delta t \widehat{u} / 2) B}{-v \alpha \Delta t A+D_{0}+\iota(\alpha \Delta t \widehat{u} / 2) B}\right|\left|\xi_{0}\right| \\
& +\left|\frac{w_{m}-\sum_{k=1}^{m-1}\left(w_{m-k+1}-w_{m-k}\right)}{-v \alpha \Delta t A+D_{0}+\iota(\alpha \Delta t \widehat{u} / 2) B}\right| \xi_{0} \mid .
\end{aligned}
$$

Using Lemma 1, we have

$$
\left|\xi_{m+1}\right| \leq \frac{\left|v \alpha \Delta t A+D_{0}-w_{1}-\iota(\alpha \Delta t \widehat{u} / 2) B\right|+w_{1}}{\left|-v \alpha \Delta t A+D_{0}+\iota(\alpha \Delta t \widehat{u} / 2) B\right|}\left|\xi_{0}\right|
$$

Consider the following two cases:

Case 3. If $v \alpha \Delta t A+D_{0}-w_{1}>0$, then, we have

$$
\left|\xi_{m+1}\right| \leq\left(\frac{v \alpha \Delta t A+D_{0}}{-v \alpha \Delta t A+D_{0}}\right)\left|\xi_{0}\right| \leq\left|\xi_{0}\right|
$$

Case 4. If $v \alpha \Delta t A+D_{0}-w_{1} \leq 0$, then, we have

$$
\left|\xi_{m+1}\right| \leq\left(\frac{2 w_{1}-v \alpha \Delta t A-D_{0}}{-v \alpha \Delta t A+D_{0}}\right)\left|\xi_{0}\right|
$$

Therefore,

$$
\frac{2 w_{1}-v \alpha \Delta t A-D_{0}}{-v \alpha \Delta t A+D_{0}} \leq 1
$$

That is, $w_{1} \leq D_{0}$, or $\left|\xi_{m+1}\right| \leq\left|\xi_{0}\right|$

By mathematical induction, we finish the proof.

Theorem 5. For $\alpha \in(0,1)$, the finite difference scheme Equation (20) is stable if $w_{1} \leq D_{0}$.

Proof. Let $w_{1} \leq D_{0}$, using Lemma 2 and Parseval's equality, we get

$$
\begin{aligned}
\left\|\rho^{n}\right\|_{2} & =\sum_{j=1}^{J-1} \sum_{i=1}^{I-1} \Delta y \Delta x\left|\rho_{i, j}^{n}\right|^{2}=\Delta y \Delta x \sum_{j=1}^{J-1} \sum_{i=1}^{I-1}\left|\xi_{n} e^{l\left(\sigma_{x} i \Delta x+\sigma_{y} j \Delta y\right)}\right|^{2} \\
& =\Delta y \Delta x \sum_{j=1}^{J-1} \sum_{i=1}^{I-1}\left|\xi_{n}\right|^{2} \leq \Delta y \Delta x \sum_{j=1}^{J-1} \sum_{i=1}^{I-1}\left|\xi_{0}\right|^{2} \\
& =\Delta y \Delta x \sum_{j=1}^{J-1} \sum_{i=1}^{I-1}\left|\xi_{0} e^{\iota\left(\sigma_{x} i \Delta x+\sigma_{y} j \Delta y\right)}\right|^{2}=\left\|\rho^{0}\right\|_{2} .
\end{aligned}
$$

So the difference scheme Equation (20) is conditionally stable.
Lemma 6. If $w_{1} \leq D_{0}$ in Equation (22) and $\alpha \in(0,1)$, then, we have

$$
\left|\eta_{n}\right| \leq\left|\eta_{0}\right|, n=1,2, \cdots, N
$$

where $\eta_{n}$ is defined in Equation (38).

Proof. Substituting $\phi_{i, j}^{n}=\eta_{n} e^{l\left(\sigma_{x} i \Delta x+\sigma_{y} j \Delta y\right)}$ into Equation (35), we have

$$
\begin{aligned}
\left(D_{0}\right. & \left.+2 r_{x x}\right) \eta_{n+1} e^{l\left(\sigma_{x} i \Delta x+\sigma_{y} j \Delta y\right)}-\frac{r_{x x}}{2} \eta_{n+1} e^{l\left(\sigma_{x}(i+1) \Delta x+\sigma_{y}(j-1) \Delta y\right)} \\
& -\frac{r_{x x}}{2} \eta_{n+1} e^{l\left(\sigma_{x}(i-1) \Delta x+\sigma_{y}(j+1) \Delta y\right)} \\
& -\left(\frac{r_{x x}}{2}-\widehat{u} r_{x}\right) \eta_{n+1} e^{l\left(\sigma_{x}(i+1) \Delta x+\sigma_{y}(j+1) \Delta y\right)} \\
& -\left(\frac{r_{x x}}{2}+\widehat{u} r_{x}\right) \eta_{n+1} e^{l\left(\sigma_{x}(i-1) \Delta x+\sigma_{y}(j-1) \Delta y\right)} \\
= & \left(D_{0}-w_{1}-2 r_{x x}\right) \eta_{n} e^{t\left(\sigma_{x} i \Delta x+\sigma_{y} j \Delta y\right)}+\frac{r_{x x}}{2} \eta_{n} e^{l\left(\sigma_{x}(i+1) \Delta x+\sigma_{y}(j-1) \Delta y\right)} \\
& +\frac{r_{x x}}{2} \eta_{n} e^{l\left(\sigma_{x}(i-1) \Delta x+\sigma_{y}(j+1) \Delta y\right)}+\left(\frac{r_{x x}}{2}-\widehat{u} r_{x}\right) \eta_{n} e^{l\left(\sigma_{x}(i+1) \Delta x+\sigma_{y}(j+1) \Delta y\right)} \\
& +\left(\frac{r_{x x}}{2}+\widehat{u} r_{x}\right) \eta_{n} e^{l\left(\sigma_{x}(i-1) \Delta x+\sigma_{y}(j-1) \Delta y\right)}+w_{n} \eta_{0} e^{l\left(\sigma_{x} i \Delta x+\sigma_{y} j \Delta y\right)} \\
& -\sum_{k=1}^{n-1}\left(w_{n-k+1}-w_{n-k}\right) \eta_{k} e^{l\left(\sigma_{x} i \Delta x+\sigma_{y} j \Delta y\right)},
\end{aligned}
$$

by simple computation and noticing that $e^{\iota \beta}+e^{-\iota \beta}=2$ $\cos (\beta)$ and $e^{\iota \beta}-e^{-\iota \beta}=2 \iota \sin (\beta)$, we can get

$$
\begin{aligned}
& \eta_{n+1}\left(D_{0}+r_{x x}(2-A)+2 \widehat{\imath} r_{x} B\right)=\eta_{n}\left(D_{0}-w_{1}-r_{x x}(2-A)-2 \widehat{\iota} r_{x} B\right) \\
& +w_{n} \eta_{0}+\sum_{k=1}^{n-1}\left(w_{n-k}-w_{n-k+1}\right) \eta_{k},
\end{aligned}
$$

where

$$
\begin{gathered}
A=\cos \left(\sigma_{x} \Delta x-\sigma_{y} \Delta y\right)+\cos \left(\sigma_{x} \Delta x+\sigma_{y} \Delta y\right), \\
B=\sin \left(\sigma_{x} \Delta x+\sigma_{y} \Delta y\right) .
\end{gathered}
$$

First, setting $n=0$ in Equation (57), we obtain

$$
\begin{aligned}
\left|\eta_{1}\right| & =\left|\frac{D_{0}-r_{x x}(2-A)-2 \widehat{\imath} r_{x} B}{D_{0}+r_{x x}(2-A)+2 \imath \widehat{u} r_{x} B}\right|\left|\eta_{0}\right| \\
& =\sqrt{\frac{\left(D_{0}-r_{x x}(2-A)\right)^{2}+\left(2 u \wedge r_{x} B\right)^{2}}{\left(D_{0}+r_{x x}(2-A)\right)^{2}+\left(2 u \wedge r_{x} B\right)^{2}}}\left|\eta_{0}\right| \leq\left|\eta_{0}\right| .
\end{aligned}
$$

Now, assume that we have proved that $\left|\eta_{n}\right| \leq\left|\eta_{0}\right|, n=1$, $2, \cdots, m$. 
We should prove this for $n=m+1$. Utilizing Equation (57), we obtain

$$
\begin{aligned}
\left|\eta_{m+1}\right| \leq & \left|\frac{D_{0}-w_{1}-r_{x x}(2-A)-2 \widehat{\imath} r_{x} B}{D_{0}+r_{x x}(2-A)+2 \imath \widehat{u} r_{x} B}\right|\left|\eta_{0}\right| \\
& +\left|\frac{w_{m}+\sum_{k=1}^{m-1}\left(w_{m-k}-w_{m-k+1}\right)}{D_{0}+r_{x x}(2-A)+2 \imath \widehat{u} r_{x} B}\right|\left|\eta_{0}\right| .
\end{aligned}
$$

According to Lemma 1, we have

$$
\left|\eta_{m+1}\right| \leq \frac{\left|D_{0}-w_{1}-r_{x x}(2-A)-2 \imath \widehat{u} r_{x} B\right|+w_{1}}{\left|D_{0}+r_{x x}(2-A)+2 \imath \widehat{u} r_{x} B\right|}\left|\eta_{0}\right| .
$$

Consider the following two cases:

Case 7. If $D_{0}-w_{1}-r_{x x}(2-A)>0$, then, we have

$$
\left|\eta_{m+1}\right| \leq\left(\frac{D_{0}-r_{x x}(2-A)}{D_{0}+r_{x x}(2-A)}\right)\left|\eta_{0}\right| \leq\left|\eta_{0}\right|
$$

Case 8. If $D_{0}-w_{1}-r_{x x}(2-A) \leq 0$, then, we have

$$
\left|\eta_{m+1}\right| \leq\left(\frac{2 w_{1}-D_{0}+r_{x x}(2-A)}{D_{0}+r_{x x}(2-A)}\right)\left|\eta_{0}\right|
$$

So that,

$$
\frac{2 w_{1}-D_{0}+r_{x x}(2-A)}{D_{0}+r_{x x}(2-A)} \leq 1
$$

This means $w_{1} \leq D_{0}$, or $\left|\eta_{m+1}\right| \leq\left|\eta_{0}\right|$.

By mathematical induction, the proof is complete.

Theorem 9. For $\alpha \in(0,1)$, the finite difference scheme Equation (22) is stable if $w_{1} \leq D_{0}$.

Proof. Suppose $w_{1} \leq D_{0}$, from Lemma 6 and Parseval's equality, we get

$$
\begin{aligned}
\left\|\phi^{n}\right\|_{2} & =\sum_{j=1}^{J-1} \sum_{i=1}^{I-1} \Delta y \Delta x\left|\phi_{i, j}^{n}\right|^{2}=\Delta y \Delta x \sum_{j=1}^{J-1} \sum_{i=1}^{I-1}\left|\eta_{n} e^{l\left(\sigma_{x} i \Delta x+\sigma_{y} j \Delta y\right)}\right|^{2} \\
& =\Delta y \Delta x \sum_{j=1}^{J-1} \sum_{i=1}^{I-1}\left|\eta_{n}\right|^{2} \leq \Delta y \Delta x \sum_{j=1}^{J-1} \sum_{i=1}^{I-1}\left|\eta_{0}\right|^{2} \\
& =\Delta y \Delta x \sum_{j=1}^{J-1} \sum_{i=1}^{I-1}\left|\eta_{0} e^{l\left(\sigma_{x} i \Delta x+\sigma_{y} j \Delta y\right)}\right|^{2}=\left\|\phi^{0}\right\|_{2} .
\end{aligned}
$$

So the difference scheme Equation (22) is conditionally stable.

\section{Convergence Analysis}

We first introduce some notations and lemmas which will be used in the convergence analysis.

$$
\begin{gathered}
\delta_{x}^{2} v_{i, j}^{n}=\frac{v_{i+1, j}^{n}-2 v_{i, j}^{n}+v_{i-1, j}^{n}}{(\Delta x)^{2}}, \delta_{y}^{2} v_{i, j}^{n}=\frac{v_{i, j+1}^{n}-2 v_{i, j}^{n}+v_{i, j-1}^{n}}{(\Delta y)^{2}}, \\
\Delta_{x}^{0} v_{i, j}^{n}=\frac{v_{i+1, j}^{n}-v_{i-1, j}^{n}}{2 \Delta x}, \Delta_{y}^{0} v_{i, j}^{n}=\frac{v_{i, j+1}^{n}-v_{i, j-1}^{n}}{2 \Delta y}, \\
(\mathbf{v}, \mathbf{w})=\Delta x \Delta y \sum_{j=1}^{J-1} \sum_{i=1}^{I-1} v_{i j} w_{i j},\|\mathbf{v}\|=\left[\Delta x \Delta y \sum_{j=1}^{J-1} \sum_{i=1}^{I-1}\left(v_{i j}\right)^{2}\right]^{1 / 2}, \\
c_{0}=\max _{(x, y, t) \in[0, L] \times[0, L] \times(0, T)}\left\{|u(x, y, t)|,\left|\frac{\partial u}{\partial x}(x, y, t)\right|,\left|\frac{\partial u}{\partial y}(x, y, t)\right|\right\} .
\end{gathered}
$$

It is straightforward to show

$$
\left|\Delta_{x}^{0} v, v\right|=0,\left|\Delta_{y}^{0} v, v\right|=0
$$

Notice that in this section we suppose $C$ stands for a positive constant independent of $\Delta t, \Delta x, \Delta y, i, j$, and $n$, which may take different values at different places.

Lemma 10. (Discrete Gronwall's inequality [29]).

Suppose $d$ be a nonnegetive constant, $\left\{z_{n}\right\}$ and $\left\{f_{n}\right\}$ are nonnegative sequences. Let

$$
z_{n} \leq d+\sum_{0 \leq k<n} f_{k} z_{k}, n \geq 0
$$

then

$$
z_{n} \leq d \exp \left(\sum_{0 \leq j<n} f_{j}\right), n \geq 0
$$

Theorem 11. The Crank-Nicolson scheme Equation (20) is convergent and the order of convergence is $\mathrm{O}\left(\Delta t+\Delta x^{2}+\Delta y^{2}\right)$.

Proof. Let $e_{i, j}^{n}$ be the error at $\left(x_{i}, y_{j}, t_{n}\right)$ as defined below

$$
\begin{aligned}
e_{i, j}^{n} & =u\left(x_{i}, y_{j}, t_{n}\right)-U_{i, j}^{n} \\
& =u_{i, j}^{n}-U_{i, j}^{n}, 1 \leq j \leq J, 1 \leq i \leq I, 1 \leq n \leq N .
\end{aligned}
$$

Substituting Equation (70) in Equation (19), we get the following error equations 
TABle 1: Absolute errors of Example 13 at $T=1$ for $\operatorname{Re}=10, \Delta t=0.01, \alpha=0.1$, and $\alpha=0.3$.

\begin{tabular}{lcccccccc}
\hline \multirow{2}{*}{$N$} & \multicolumn{2}{c}{$\alpha=0.1$} & Time & EDG & Time & CN & Time & EDG \\
\hline 9 & $4.2593 e-03$ & 0.75 & $6.1387 e-03$ & 0.74 & $3.9001 e-03$ & 0.22 & $6.0143 e-03$ & 0.11 \\
19 & $9.8379 e-04$ & 0.93 & $1.6263 e-03$ & 0.69 & $9.0356 e-04$ & 1.01 & $1.6124 e-03$ & 0.51 \\
49 & $1.9672 e-04$ & 9.82 & $2.6073 e-04$ & 4.69 & $1.8465 e-04$ & 10.61 & $2.6040 e-04$ & 4.73 \\
99 & $9.1297 e-05$ & 123.15 & $9.7575 e-05$ & 39.87 & $8.8260 e-05$ & 118.02 & $9.3869 e-05$ & 42.01 \\
\hline
\end{tabular}

TABle 2: Absolute errors of Example 13 at $T=1$ for $\operatorname{Re}=10, \Delta t=0.01, \alpha=0.7$, and $\alpha=0.9$.

\begin{tabular}{|c|c|c|c|c|c|c|c|c|}
\hline \multirow{2}{*}{$N$} & \multicolumn{4}{|c|}{$\alpha=0.7$} & \multicolumn{4}{|c|}{$\alpha=0.9$} \\
\hline & $\mathrm{CN}$ & Time & EDG & Time & $\mathrm{CN}$ & Time & EDG & Time \\
\hline 9 & $2.7474 e-03$ & 0.16 & $5.6163 e-03$ & 0.12 & $1.8409 e-03$ & 0.17 & $5.2940 e-03$ & 0.12 \\
\hline 19 & $6.6801 e-04$ & 0.84 & $1.5736 e-03$ & 0.60 & $4.8985 e-04$ & 0.86 & $1.5707 e-03$ & 0.59 \\
\hline 49 & $1.5163 e-04$ & 10.26 & $2.5934 e-04$ & 5.68 & $1.4882 e-04$ & 10.38 & $2.5869 e-04$ & 5.35 \\
\hline 99 & $8.2965 e-05$ & 119.25 & $8.6473 e-05$ & 41.44 & $1.0295 e-04$ & 121.48 & $1.0451 e-04$ & 42.37 \\
\hline
\end{tabular}

$$
\begin{aligned}
-w_{n} e_{i j}^{0}+ & \sum_{k=1}^{n-1}\left(w_{n-k+1}-w_{n-k}\right) e_{i j}^{k}+w_{1} e_{i j}^{n}+\left(e_{i j}^{n+1}-e_{i j}^{n}\right) D_{0} \\
= & \frac{v \alpha \Delta t}{2}\left(\delta_{x}^{2}\left(e_{i, j}^{n+1}+e_{i, j}^{n}\right)+\delta_{y}^{2}\left(e_{i, j}^{n+1}+e_{i, j}^{n}\right)\right) \\
& -\frac{\alpha \Delta t}{2}\left(\sigma_{4}+\sigma_{5}\right)+\alpha \Delta t R_{i, j}^{n+\frac{1}{2}} \\
1 \leq & j \leq J-1,1 \leq i \leq I-1,1 \leq n \leq N, \\
e_{0, j}^{n}= & e_{I, j}^{n}=0,1 \leq n \leq N, 0 \leq j \leq J, \\
e_{i, 0}^{n}= & e_{i, j}^{n}=0,1 \leq n \leq N, 0 \leq i \leq I, \\
e_{i, j}^{0}= & 0,0 \leq j \leq J, 1 \leq i \leq I,
\end{aligned}
$$

where

$$
\begin{aligned}
R_{i, j}^{n+\frac{1}{2}} & =O\left(\Delta t+\Delta x^{2}+\Delta y^{2}\right), \\
\sigma_{4} & =e_{i, j}^{n} \Delta_{x}^{0} u_{i, j}^{n+1}+u_{i, j}^{n} \Delta_{x}^{0} e_{i, j}^{n+1}+e_{i, j}^{n+1} \Delta_{x}^{0} u_{i, j}^{n}+u_{i, j}^{n+1} \Delta_{x}^{0} e_{i, j}^{n}, \\
\sigma_{5} & =e_{i, j}^{n} \Delta_{y}^{0} u_{i, j}^{n+1}+u_{i, j}^{n} \Delta_{y}^{0} e_{i, j}^{n+1}+e_{i, j}^{n+1} \Delta_{y}^{0} u_{i, j}^{n}+u_{i, j}^{n+1} \Delta_{y}^{0} e_{i, j}^{n} .
\end{aligned}
$$

Multiplying Equation (71) by $\Delta x \Delta y\left(e_{i, j}^{n+1}+e_{i, j}^{n}\right)$ and summing up for $i$ from 1 to $\mathrm{I}-1$ and $j$ from 1 to $J-1$, we obtain

$$
\begin{aligned}
\left|\sum_{k=1}^{n-1}\left(w_{n-k+1}-w_{n-k}\right) e^{k}+w_{1} e^{n}+\left(e^{n+1}-e^{n}\right) D_{0}, e^{n+1}+e^{n}\right| & v \alpha \Delta t \\
= & \frac{v \Delta}{2}\left(\left|\delta_{x}^{2}\left(e^{n+1}+e^{n}\right), e^{n+1}+e^{n}\right|+\left|\delta_{y}^{2}\left(e^{n+1}+e^{n}\right), e^{n+1}+e^{n}\right|\right) \\
& -\frac{\alpha \Delta t}{2}\left|\sigma_{4}+\sigma_{5}, e^{n+1}+e^{n}\right|+\alpha \Delta t\left|R^{n+\frac{1}{2}}, e^{n+1}+e^{n}\right|, 1 \leq n \leq N .
\end{aligned}
$$

It is clear that $\left|\delta_{x}^{2}\left(e^{n+1}+e^{n}\right), e^{n+1}+e^{n}\right| \leq 0$ and $\mid \delta_{y}^{2}\left(e^{n+1}\right.$ $\left.+e^{n}\right), e^{n+1}+e^{n} \mid \leq 0$, so we have

$$
\begin{aligned}
& \left.D_{0}\left\|e^{n+1}\right\|\right|^{2} \leq\left(D_{0}-w_{1}\right)\left\|e^{n}\right\|^{2}+w_{1}|| e^{n+1}, e^{n} \| \\
& +\left|\sum_{k=1}^{n-1}\left(w_{n-k}-w_{n-k+1}\right) e^{k}, e^{n+1}+e^{n}\right| \\
& +\frac{\alpha \Delta t}{2}|| \sigma_{4}+\sigma_{5}, e^{n+1}+e^{n} \| \\
& +\alpha \Delta t\left|R^{n+\frac{1}{2}}, e^{n+1}+e^{n}\right|, 1 \leq n \leq N .
\end{aligned}
$$

Now, we estimate the third, fourth, and fifth terms of the right-hand side of Equation (74), respectively

$$
\begin{aligned}
& \left|\sum_{k=1}^{n-1}\left(w_{n-k}-w_{n-k+1}\right) e^{k}, e^{n+1}+e^{n}\right| \\
& \quad=\sum_{k=1}^{n-1}\left(w_{n-k}-w_{n-k+1}\right)\left|e^{k}, e^{n+1}\right|+\sum_{k=1}^{n-1}\left(w_{n-k}-w_{n-k+1}\right)\left|e^{k}, e^{n}\right| .
\end{aligned}
$$

Using Young inequality $a b \leq \varepsilon a^{2}+(1 / 4 \varepsilon) b^{2}, a, b \in \mathbb{R}$, and Lemma 1, we have

$$
\begin{aligned}
& \left|\sum_{k=1}^{n-1}\left(w_{n-k}-w_{n-k+1}\right) e^{k}, e^{n+1}+e^{n}\right| \leq C \Delta t^{2} \sum_{k=1}^{n-1}\left\|e^{k}\right\|^{2} \\
& \quad+C \Delta t\left(\left\|e^{n}\right\|^{2}+\left\|e^{n+1}\right\|^{2}\right) .
\end{aligned}
$$

For the fourth term, utilizing Young inequality and Equation (67), we obtain 


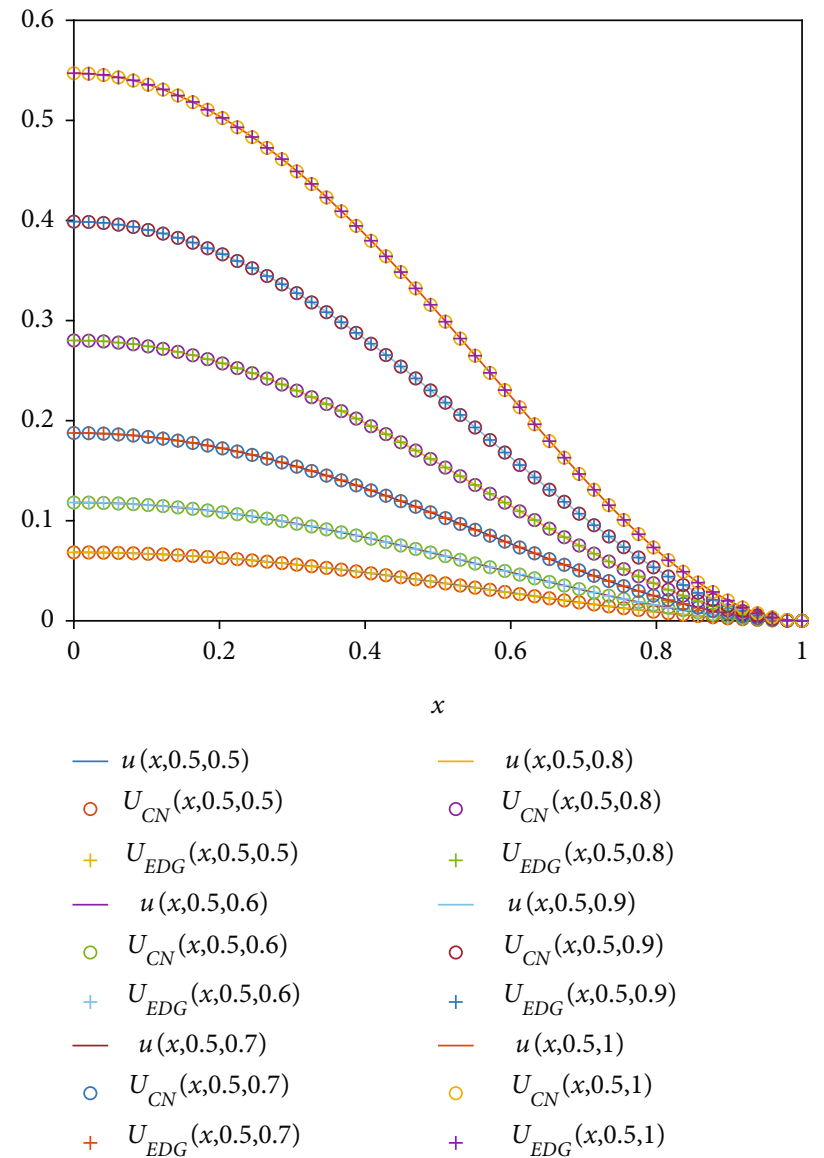

(a)

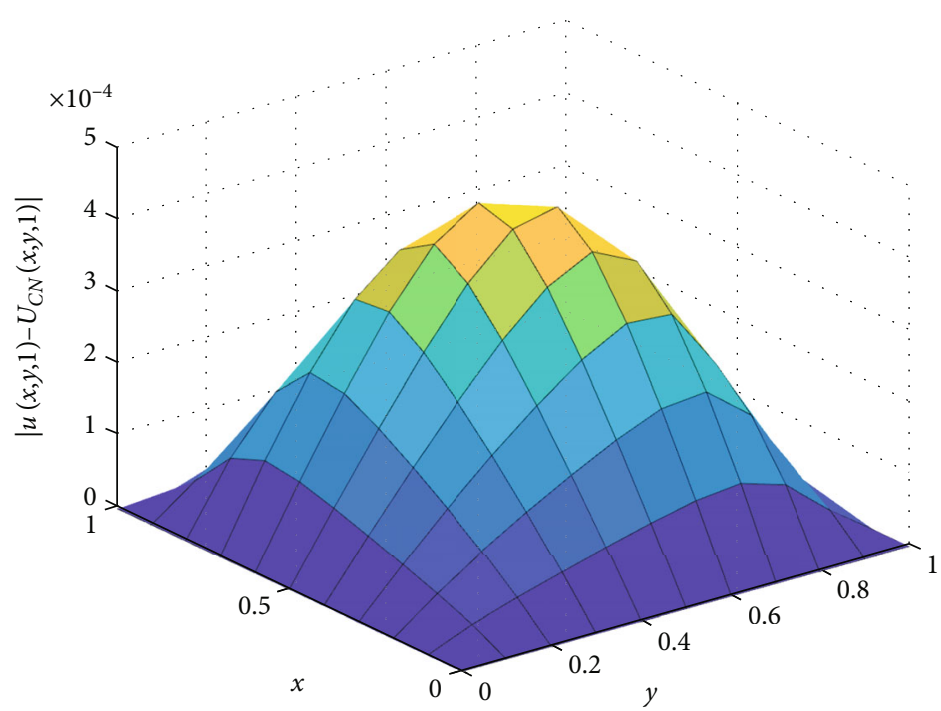

(b)

Figure 3: The exact, Crank-Nicolson, and EDG solutions in different values of $t$ and $y=0.5$ (a) and the absolute error of Crank-Nicolson method at $T=1$ (b) of Example 13 for $\Delta t=0.01, \operatorname{Re}=100, N=49$, and $\alpha=0.5$. 


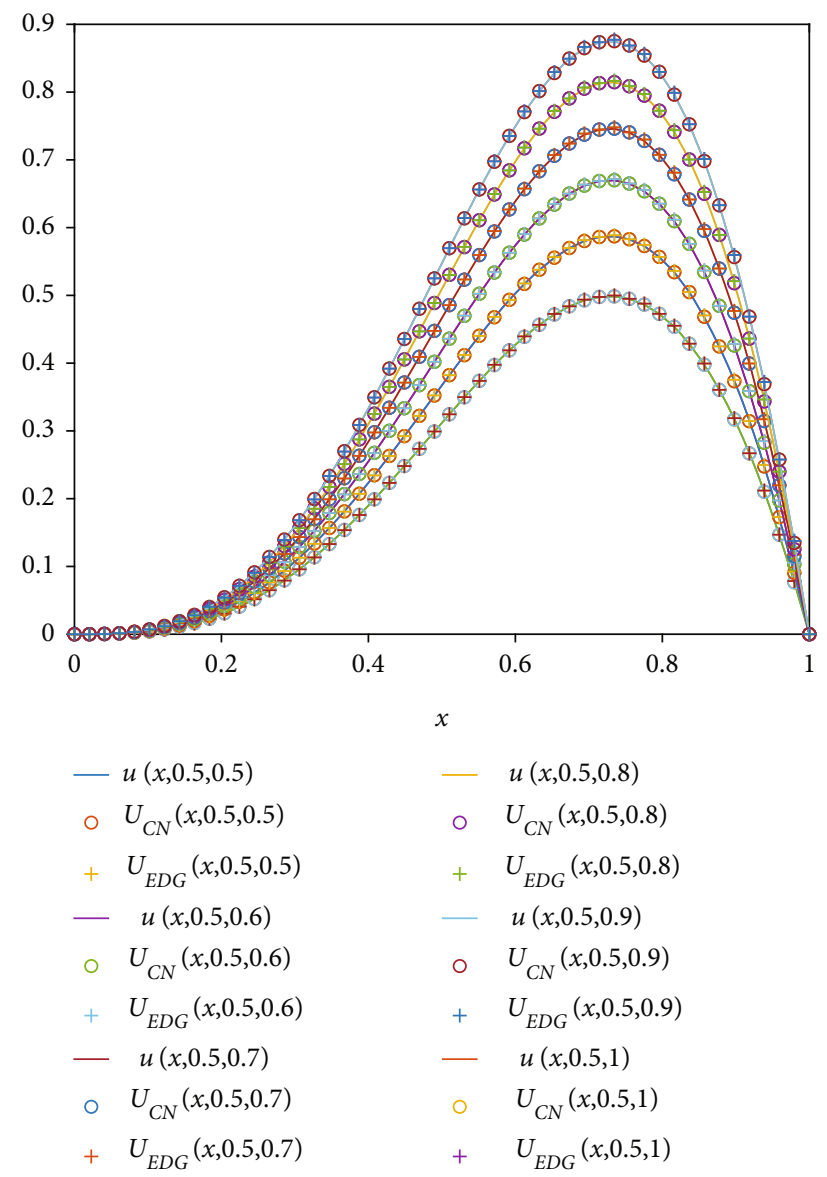

(a)

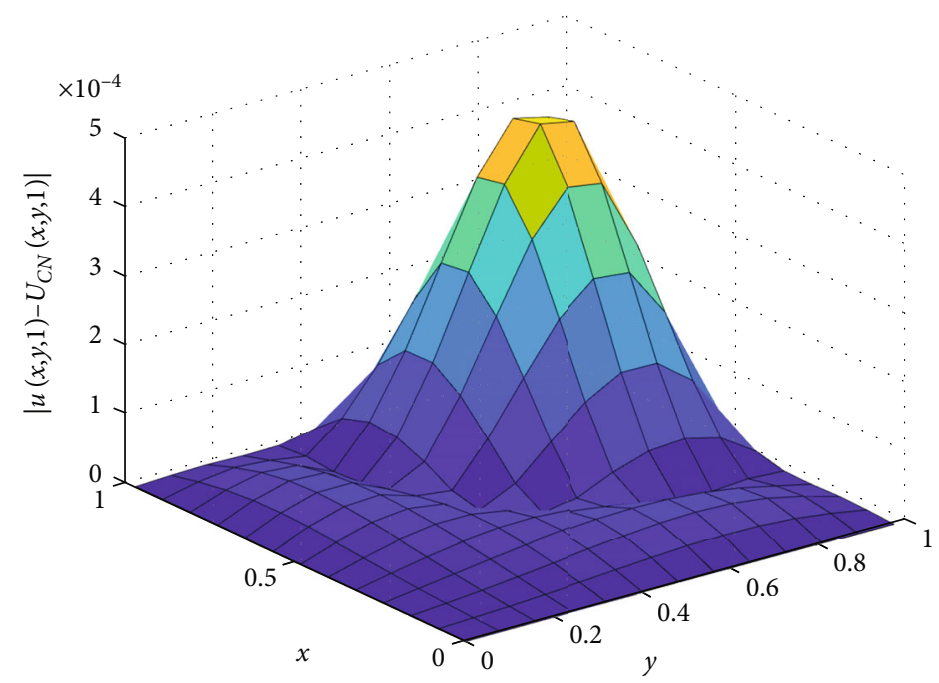

(b)

Figure 4: The exact, Crank-Nicolson and EDG solutions in different values of $t$ and $y=0.5$ (a) and the absolute error of Crank-Nicolson method at $T=1$ (b) of Example 14 for $\Delta t=0.01, \operatorname{Re}=50, N=49$, and $\alpha=0.5$. 
TABle 3: Absolute errors of Example 14 at $T=1$ for $\operatorname{Re}=100, \Delta t=0.01, \alpha=0.1$, and $\alpha=0.3$.

\begin{tabular}{|c|c|c|c|c|c|c|c|c|}
\hline \multirow{2}{*}{$N$} & \multicolumn{4}{|c|}{$\alpha=0.1$} & \multicolumn{4}{|c|}{$\alpha=0.3$} \\
\hline & $\mathrm{CN}$ & Time & EDG & Time & $\mathrm{CN}$ & Time & EDG & Time \\
\hline 9 & $4.1318 e-03$ & 0.40 & $1.0556 e-02$ & 0.26 & $3.7717 e-03$ & 0.53 & $9.8104 e-03$ & 0.28 \\
\hline 19 & $8.8813 e-04$ & 1.04 & $2.6531 e-03$ & 0.73 & $8.1156 e-04$ & 1.17 & $2.5458 e-03$ & 0.73 \\
\hline 49 & $1.3364 e-04$ & 12.14 & $4.1507 e-04$ & 4.81 & $1.2247 e-04$ & 15.24 & $4.0109 e-04$ & 4.86 \\
\hline 99 & $3.4588 e-05$ & 122.31 & $1.0389 e-04$ & 40.55 & $3.1779 e-05$ & 130.36 & $1.0045 e-04$ & 40.71 \\
\hline
\end{tabular}

TABle 4: Absolute errors of Example 14 at $T=1$ for $\operatorname{Re}=100, \Delta t=0.01, \alpha=0.7$, and $\alpha=0.9$.

\begin{tabular}{|c|c|c|c|c|c|c|c|c|}
\hline \multirow{2}{*}{$N$} & \multicolumn{4}{|c|}{$\alpha=0.7$} & \multicolumn{4}{|c|}{$\alpha=0.9$} \\
\hline & $\mathrm{CN}$ & Time & EDG & Time & $\mathrm{CN}$ & Time & EDG & Time \\
\hline 9 & $2.9459 e-03$ & 0.57 & $8.2550 e-03$ & 0.34 & $2.4730 e-03$ & 0.96 & $7.5148 e-03$ & 0.26 \\
\hline 19 & $6.3611 e-04$ & 1.39 & $2.3322 e-03$ & 0.84 & $5.4346 e-04$ & 1.56 & $2.2536 e-03$ & 0.84 \\
\hline 49 & $9.7981 e-05$ & 15.19 & $3.7198 e-04$ & 5.69 & $8.6200 e-05$ & 17.26 & $3.5924 e-04$ & 5.32 \\
\hline 99 & $2.5774 e-05$ & 126.65 & $9.3273 e-05$ & 44.77 & $2.4146 e-05$ & 136.80 & $9.1092 e-05$ & 43.01 \\
\hline
\end{tabular}

TABle 5: Absolute errors of Example 15 at $T=1$ for $\operatorname{Re}=5, \Delta t=0.01, \alpha=0.1$, and $\alpha=0.3$.

\begin{tabular}{|c|c|c|c|c|c|c|c|c|}
\hline \multirow{2}{*}{$N$} & \multicolumn{4}{|c|}{$\alpha=0.1$} & \multicolumn{4}{|c|}{$\alpha=0.3$} \\
\hline & $\mathrm{CN}$ & Time & EDG & Time & $\mathrm{CN}$ & Time & EDG & Time \\
\hline 9 & $2.1509-03$ & 0.51 & $7.1351 e-03$ & 0.24 & $2.0937 e-03$ & 0.19 & $6.9914 e-03$ & 0.12 \\
\hline 19 & $4.8726 e-04$ & 1.09 & $1.7356 e-03$ & 0.59 & $4.7437 e-04$ & 0.91 & $1.7078 e-03$ & 0.56 \\
\hline 49 & $7.3239 e-05$ & 11.88 & $2.6457 e-04$ & 4.86 & $7.1213 e-05$ & 11.80 & $2.6032 e-04$ & 5.04 \\
\hline 99 & $1.7989 e-05$ & 121.06 & $6.4817 e-05$ & 40.01 & $1.7399 e-05$ & 120.95 & $6.3688 e-05$ & 40.27 \\
\hline
\end{tabular}

TABle 6: Absolute errors of Example 15 at $T=1$ for $\operatorname{Re}=5, \Delta t=0.01, \alpha=0.7$, and $\alpha=0.9$.

\begin{tabular}{|c|c|c|c|c|c|c|c|c|}
\hline \multirow{2}{*}{$N$} & \multicolumn{4}{|c|}{$\alpha=0.7$} & \multicolumn{4}{|c|}{$\alpha=0.9$} \\
\hline & $\mathrm{CN}$ & Time & EDG & Time & $\mathrm{CN}$ & Time & EDG & Time \\
\hline 9 & $1.9317 e-03$ & 0.21 & $6.5894 e-03$ & 0.12 & $1.8282 e-03$ & 0.23 & $6.3412 e-03$ & 0.13 \\
\hline 19 & $4.3742 e-04$ & 0.97 & $1.6292 e-03$ & 0.60 & $4.1260 e-04$ & 1.01 & $1.5796 e-03$ & 0.61 \\
\hline 49 & $6.4857 e-05$ & 12.21 & $2.4776 e-04$ & 5.29 & $5.9238 e-05$ & 12.58 & $2.3834 e-04$ & 5.35 \\
\hline 99 & $1.5095 e-05$ & 123.57 & $5.9878 e-05$ & 41.38 & $1.2086 e-05$ & 124.23 & $5.5847 e-05$ & 41.61 \\
\hline
\end{tabular}

$$
\begin{aligned}
& \left|\left\langle\sigma_{4}, e^{n+1}+e^{n}\right\rangle\right| \leq c_{0}\left(1+\varepsilon_{1}+\varepsilon_{2}+\varepsilon_{3}+\varepsilon_{4}+\varepsilon_{5}\right)\left\|e^{n+1}\right\|^{2} \\
& +c_{0}\left(1+\frac{1}{4 \varepsilon_{1}}+\frac{1}{4 \varepsilon_{2}}+\frac{1}{4 \varepsilon_{3}}+\frac{1}{4 \varepsilon_{4}}+\frac{1}{4 \varepsilon_{5}}\right)\left\|e^{n}\right\|^{2}
\end{aligned}
$$

Similarly, we get

$$
\begin{gathered}
\left|\left\langle\sigma_{5}, e^{n+1}+e^{n}\right\rangle\right| \leq c_{0}\left(1+\varepsilon_{6}+\varepsilon_{7}+\varepsilon_{8}+\varepsilon_{9}+\varepsilon_{10}\right)\left\|e^{n+1}\right\|^{2} \\
+c_{0}\left(1+\frac{1}{4 \varepsilon_{6}}+\frac{1}{4 \varepsilon_{7}}+\frac{1}{4 \varepsilon_{8}}+\frac{1}{4 \varepsilon_{9}}+\frac{1}{4 \varepsilon_{10}}\right)\left\|e^{n}\right\|^{2}
\end{gathered}
$$

Also for the fifth term, we have

$$
\left|R^{n+\frac{1}{2}}, e^{n+1}+e^{n}\right| \leq\left(\varepsilon_{11}+\frac{1}{4 \varepsilon_{12}}\right)\left\|R^{n+\frac{1}{2}}\right\|^{2}+\varepsilon_{12}\left\|e^{n+1}\right\|^{2}+\frac{1}{4 \varepsilon_{11}}\left\|e^{n}\right\|^{2} .
$$

For the second term on the right-hand side of Equation (74), we get

$$
w_{1}\left\|e^{n+1}, e^{n}\right\| \leq w_{1}\left(\varepsilon_{13}\left\|e^{n+1}\right\|^{2}+\frac{1}{4 \varepsilon_{13}}\left\|e^{n}\right\|^{2}\right) .
$$

Combining Equations (76)-(80) in Equation (74) and using Lemma 1, we obtain 


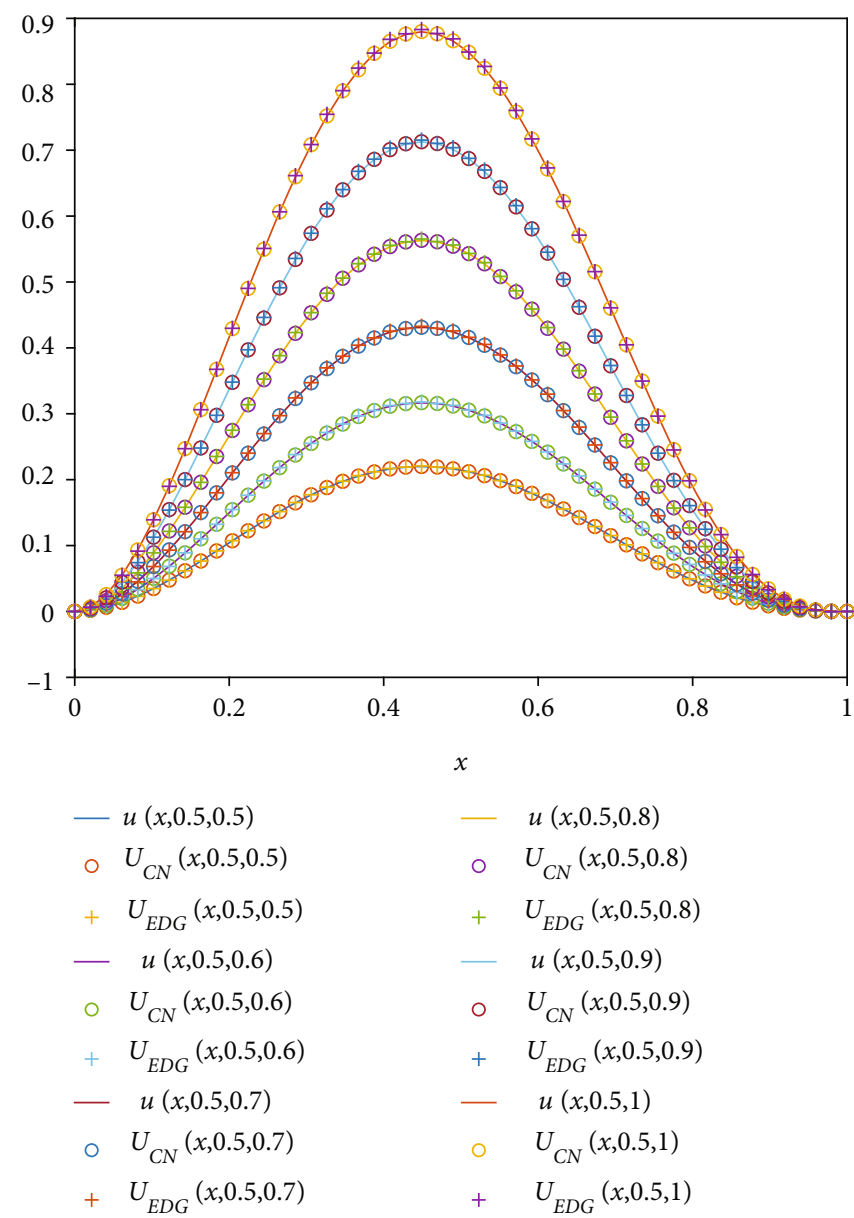

(a)

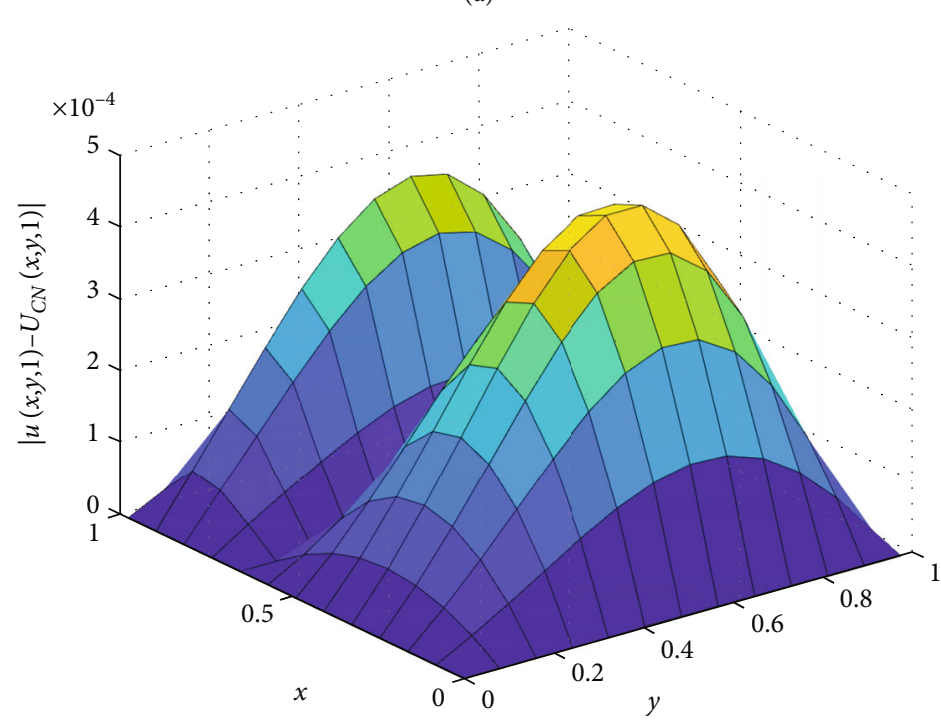

(b)

Figure 5: The exact, Crank-Nicolson and EDG solutions in different values of $t$ and $y=0.5$ (a) and the absolute error of Crank-Nicolson method at $T=1$ (b) of Example 15 for $\Delta t=0.01, \operatorname{Re}=20, N=49$, and $\alpha=0.5$. 
TABle 7: Absolute errors of Example 16 at $T=1$ for $\operatorname{Re}=2, \Delta t=0.01, \alpha=0.2$, and $\alpha=0.4$.

\begin{tabular}{lcccccccc}
\hline \multirow{2}{*}{$N$} & \multicolumn{2}{c}{$\alpha=0.2$} & \multicolumn{2}{c}{$\alpha=0.4$} & \multicolumn{2}{c}{ CN } & Time & EDG \\
\hline 9 & $2.5900 e-03$ & 0.21 & $2.1227 e-02$ & 0.19 & $2.5752 e-03$ & 0.20 & $2.1177 e-02$ & 0.18 \\
19 & $5.9920 e-04$ & 0.83 & $5.3687 e-03$ & 0.44 & $5.9600 e-04$ & 0.84 & $5.3597 e-03$ & 0.59 \\
49 & $9.3436 e-05$ & 9.41 & $8.2492 e-04$ & 5.19 & $9.2993 e-05$ & 9.70 & $8.2383 e-04$ & 5.12 \\
99 & $2.5225 e-05$ & 117.52 & $2.0397 e-04$ & 39.94 & $2.5145 e-05$ & 119.21 & $2.0376 e-04$ & 39.86 \\
\hline
\end{tabular}

TABle 8: Absolute errors of Example 16 at $T=1$ for $\operatorname{Re}=2, \Delta t=0.01, \alpha=0.6$, and $\alpha=0.8$.

\begin{tabular}{lcccccccc}
\hline \multirow{2}{N}{} & \multicolumn{2}{c}{$\alpha=0.6$} & Time & EDG & Time & CN & Time & EDG \\
\hline 9 & $2.5546 e-03$ & 0.22 & $2.1112 e-02$ & 0.20 & $2.5253 e-03$ & 0.24 & $2.1039 e-02$ & 0.22 \\
19 & $5.9150 e-04$ & 0.84 & $5.3476 e-03$ & 0.60 & $5.8469 e-04$ & 0.88 & $5.3318 e-03$ & 0.62 \\
49 & $9.2340 e-05$ & 9.88 & $8.2234 e-04$ & 5.05 & $9.0870 e-05$ & 10.19 & $8.2010 e-04$ & 5.51 \\
99 & $2.5001 e-05$ & 119.14 & $2.0348 e-04$ & 41.28 & $2.4238 e-05$ & 123.96 & $2.0280 e-04$ & 41.67 \\
\hline
\end{tabular}

$$
\begin{aligned}
D_{0}\left\|e^{n+1}\right\|^{2} \leq & C \Delta t^{2} \sum_{k=1}^{n-1}\left\|e^{k}\right\|^{2}+\Delta t\left(\lambda_{1}\left\|e^{n+1}\right\|^{2}+\lambda_{2}\left\|e^{n}\right\|^{2}\right) \\
& +\alpha \Delta t\left(\varepsilon_{11}+\frac{1}{4 \varepsilon_{12}}\right)\left\|R^{n+1 / 2}\right\|^{2}, 1 \leq n \leq N
\end{aligned}
$$

where

$$
\begin{aligned}
\lambda_{1}= & \frac{\alpha c_{0}}{2}\left(2+\varepsilon_{1}+\varepsilon_{2}+\varepsilon_{3}+\varepsilon_{4}+\varepsilon_{5}+\varepsilon_{6}+\varepsilon_{7}+\varepsilon_{8}+\varepsilon_{9}+\varepsilon_{10}\right) \\
& +\alpha \varepsilon_{12}+C \varepsilon_{13}+C \\
\lambda_{2}= & \frac{\alpha c_{0}}{2}\left(2+\frac{1}{4 \varepsilon_{1}}+\frac{1}{4 \varepsilon_{2}}+\frac{1}{4 \varepsilon_{3}}+\frac{1}{4 \varepsilon_{4}}+\frac{1}{4 \varepsilon_{5}}+\frac{1}{4 \varepsilon_{6}}+\frac{1}{4 \varepsilon_{7}}\right. \\
& \left.+\frac{1}{4 \varepsilon_{8}}+\frac{1}{4 \varepsilon_{9}}+\frac{1}{4 \varepsilon_{10}}\right)+\frac{C}{4 \varepsilon_{13}}+\frac{\alpha}{4 \varepsilon_{11}}+2 C .
\end{aligned}
$$

Therefore, we get

$$
\begin{aligned}
\left(C-\lambda_{1}\right)\left\|e^{n+1}\right\|^{2} \leq & C \Delta t \sum_{k=1}^{n-1}\left\|e^{k}\right\|^{2}+\lambda_{2}\left\|e^{n}\right\|^{2} \\
& +\alpha\left(\varepsilon_{11}+\frac{1}{4 \varepsilon_{12}}\right)\left\|R^{n+1 / 2}\right\|^{2}, 1 \leq n \leq N .
\end{aligned}
$$

In the definition of $\lambda_{1}$, we choose epsilons which $C-\lambda_{1}$ be positive, so we have

$$
\left\|e^{n+1}\right\|^{2} \leq C \Delta t \sum_{k=1}^{n-1}\left\|e^{k}\right\|^{2}+C\left\|e^{n}\right\|^{2}+\alpha C\left\|R^{n+1 / 2}\right\|^{2}, 1 \leq n \leq N .
$$

Using Lemma 10 and $n \Delta t=T$, we get

$$
\left\|e^{n+1}\right\|^{2} \leq C\left\|R^{n+1 / 2}\right\|^{2} \leq O\left(\Delta t+\Delta x^{2}+\Delta y^{2}\right), 1 \leq n \leq N,
$$

and this completes the proof.

Theorem 12. The EDG method Equation (22) is convergent and the order of convergence is $O\left(\Delta t+\Delta x^{2}+\Delta y^{2}\right)$.

Proof. The proof is similar to Theorem 11.

\section{Numerical Results}

In this section, some numerical examples are considered to demonstrate the efficiency and accuracy of the proposed methods.

In numerical examples, we suppose that $u(x, t), U_{\mathrm{CN}}(x$, $t)$, and $U_{\mathrm{EDG}}(x, t)$ denote the exact, Crank-Nicolson, and the EDG solution, respectively. Also in all Tables, the $\mathrm{CN}$ is an abbreviation for Crank-Nicolson Method.

The results obtained in this study show that the suggested methods have excellent stability, and they have verified the validity and effectiveness of the presented methods. Notably, we perform all of the computations by MATLAB R2019a software on a 64 -bit $\mathrm{PC}$ with $2.30 \mathrm{GHz}$ processor and $8 \mathrm{~GB}$ memory.

Example 13. Consider Equation (1) with the exact solution

$$
u(x, y, t)=t^{3}\left(1-x^{2}\right)^{2}\left(1-y^{2}\right)^{2}, 0 \leq x, y, t \leq 1 .
$$

In Tables 1 and 2, the maximum of absolute errors and CPU Times for Crank-Nicolson and EDG methods for $\alpha=$ $0.1,0.3$, and $\alpha=0.7,0.9$ with $T=1, \Delta t=0.01, \operatorname{Re}=10$, and different values of $N$ are tabulated, respectively. These results confirm the convergent results. In Figure 3(a), the exact, Crank-Nicolson, and EDG solutions for $y=0.5, \mathrm{Re}=100$, 


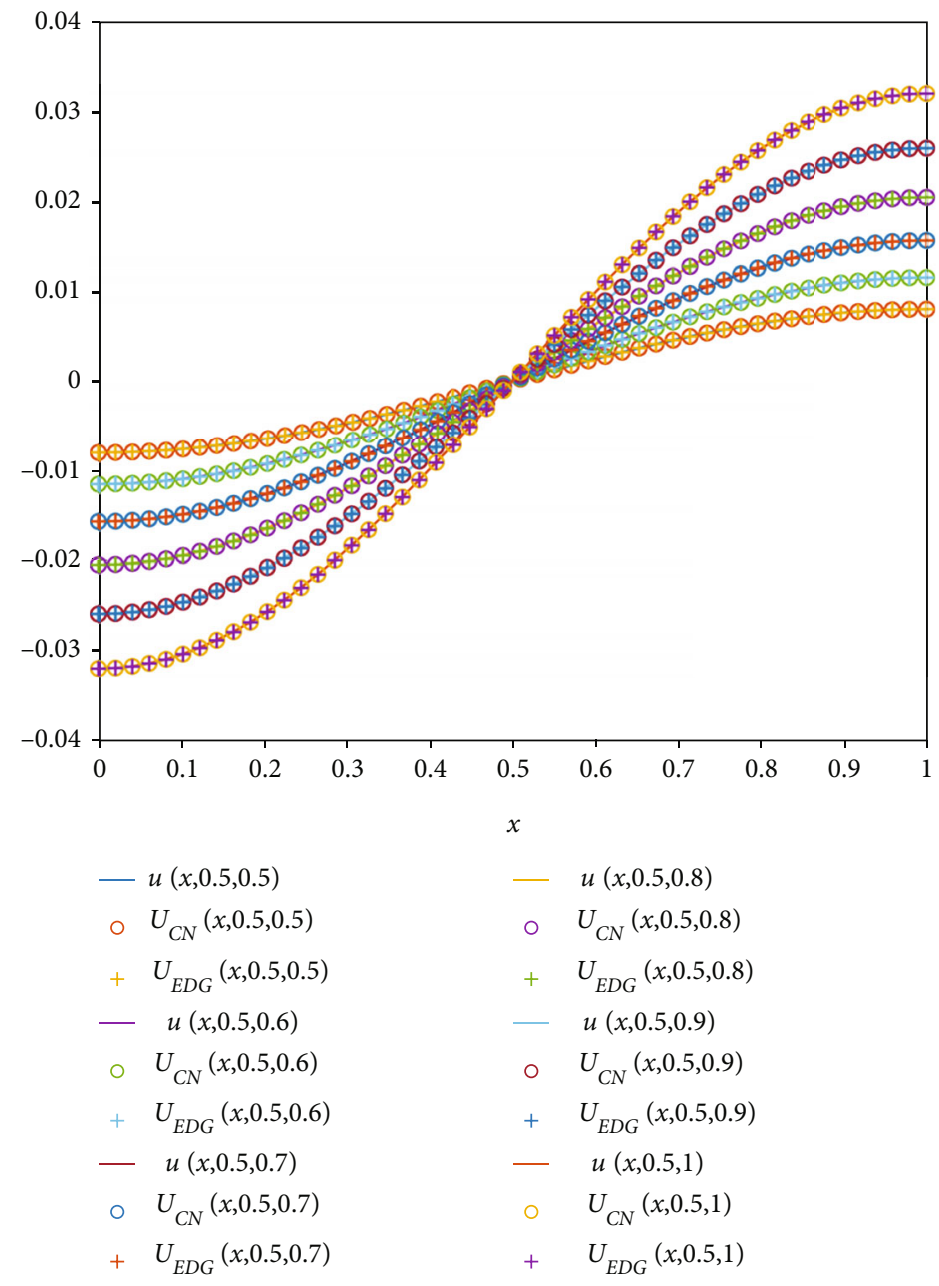

(a)

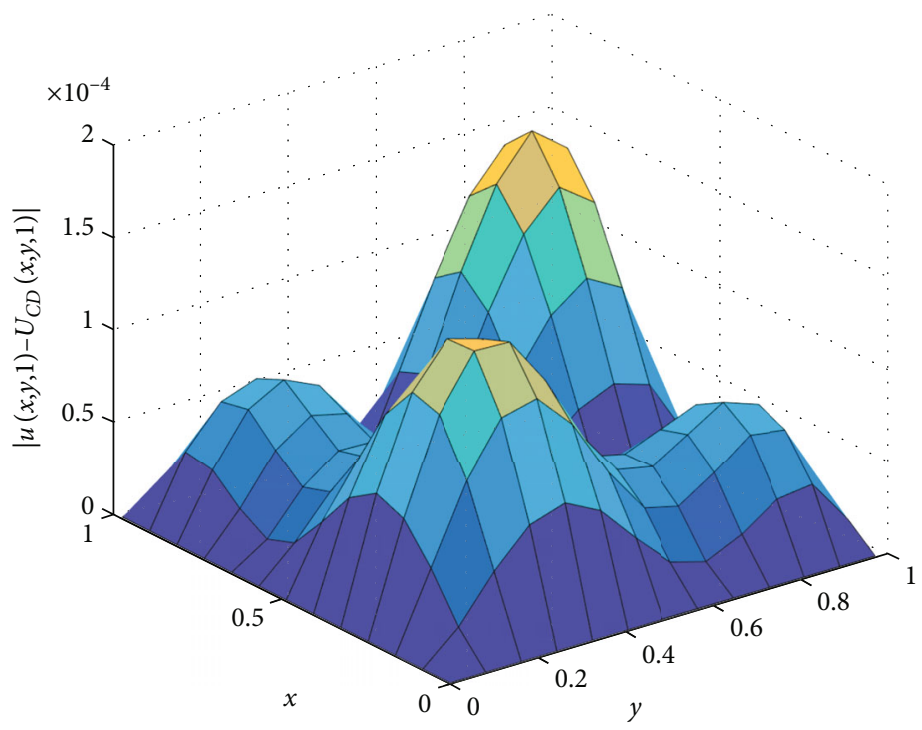

(b)

Figure 6: The exact, Crank-Nicolson, and EDG solutions in different values of $t$ (a) and the absolute error of Crank-Nicolson method at $T=1$ (b) of Example 16 for $\Delta t=0.01, \operatorname{Re}=10, N=49$, and $\alpha=0.5$. 


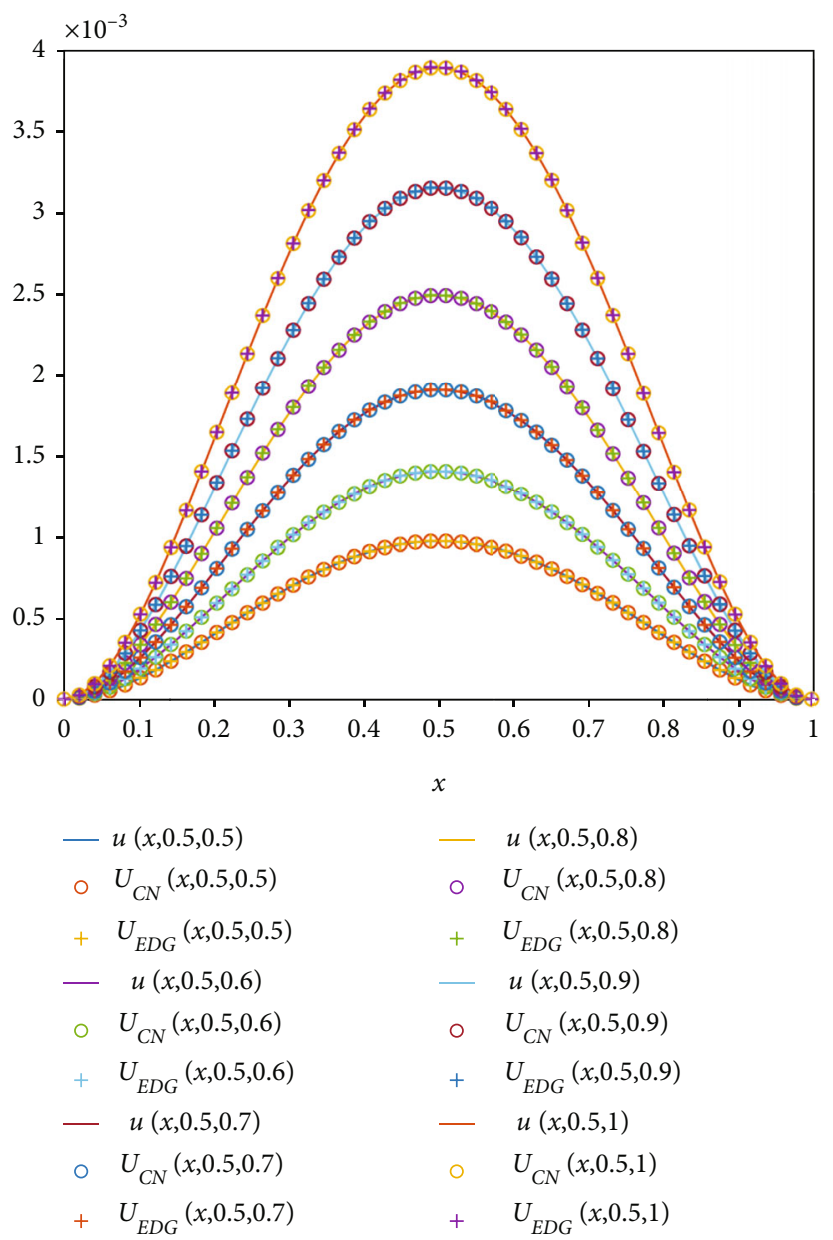

(a)

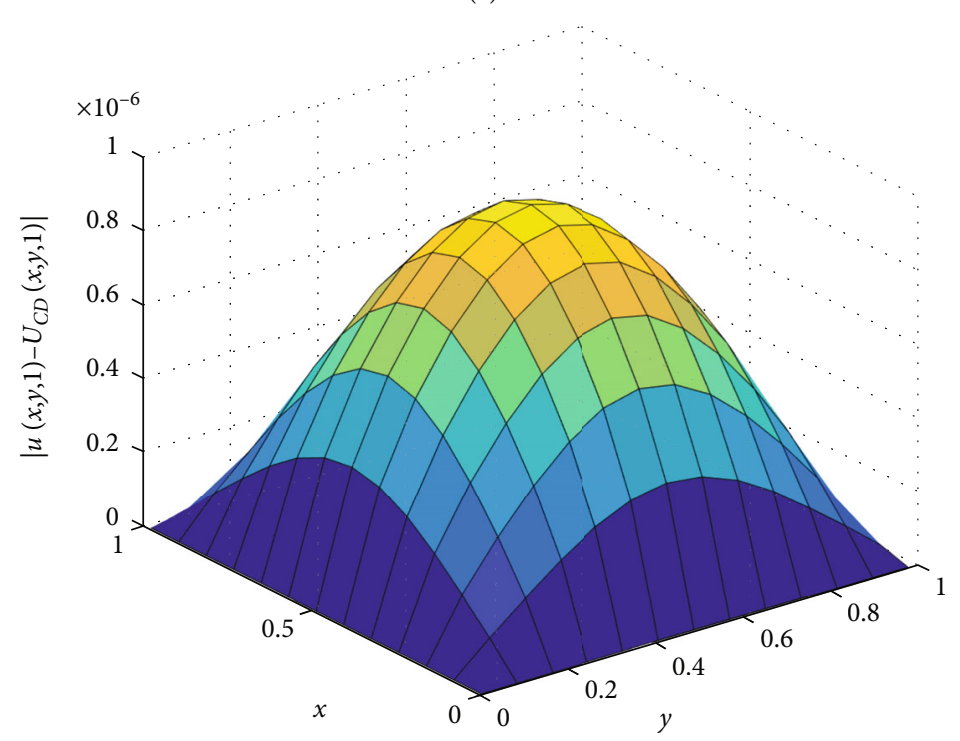

(b)

Figure 7: The exact, Crank-Nicolson and EDG solutions in different values of $t$ (a) and the absolute error of Crank-Nicolson method at $T=1$ (b) of Example 16 for $\Delta t=0.01, \operatorname{Re}=60, N=49$, and $\alpha=0.5$. 
TABLE 9: Absolute errors of Example 17 at $T=1$ for $\operatorname{Re}=100, \Delta t=0.01, \alpha=0.2$, and $\alpha=0.4$.

\begin{tabular}{|c|c|c|c|c|c|c|c|c|}
\hline \multirow{2}{*}{$N$} & \multicolumn{4}{|c|}{$\alpha=0.2$} & \multicolumn{4}{|c|}{$\alpha=0.4$} \\
\hline & $\mathrm{CN}$ & Time & EDG & Time & $\mathrm{CN}$ & Time & EDG & Time \\
\hline 9 & $2.3544 e-05$ & 0.37 & $1.2078 e-04$ & 0.15 & $2.0308 e-05$ & 0.32 & $1.1028 e-04$ & 0.14 \\
\hline 19 & $5.3089 e-06$ & 0.89 & $3.7501 e-05$ & 0.55 & $4.5547 e-06$ & 0.90 & $3.5462 e-05$ & 0.73 \\
\hline 49 & $7.8572 e-07$ & 9.70 & $6.1862 e-06$ & 4.84 & $6.5077 e-07$ & 9.72 & $5.9056 e-06$ & 5.23 \\
\hline 99 & $1.9520 e-07$ & 117.73 & $1.5238 e-06$ & 39.65 & $1.9513 e-07$ & 118.38 & $1.4377 e-06$ & 40.22 \\
\hline
\end{tabular}

TABle 10: Absolute errors of Example 17 at $T=1$ for $\operatorname{Re}=100, \Delta t=0.01, \alpha=0.6$, and $\alpha=0.8$.

\begin{tabular}{lcccccccc}
\hline \multirow{2}{N}{} & \multicolumn{2}{c}{$\alpha=0.6$} & Time & EDG & Time & CN & Time & EDG \\
\hline 9 & $1.6919 e-05$ & 0.32 & $9.8582 e-05$ & 0.26 & $1.3331 e-05$ & 0.37 & $8.5506 e-05$ & 0.27 \\
19 & $3.7515 e-06$ & 0.89 & $3.3183 e-05$ & 0.82 & $2.8606 e-06$ & 0.94 & $3.0637 e-05$ & 0.79 \\
49 & $4.9131 e-07$ & 9.91 & $5.5845 e-06$ & 5.41 & $2.7513 e-07$ & 10.06 & $5.1849 e-06$ & 5.50 \\
99 & $1.9501 e-07$ & 118.22 & $1.3268 e-06$ & 41.16 & $1.9462 e-07$ & 119 & $1.1529 e-06$ & 41.91 \\
\hline
\end{tabular}

$N=49$, and $\Delta t=0.01$ in different values of $t$ are illustrated. Furthermore in Figure 3(b), the absolute error at $T=1$ for $\operatorname{Re}=100, N=49, \Delta t=0.01$, and $\alpha=0.5$ is portrayed. According to the Figures, we can see that our numerical solutions correspond to the exact solutions.

Example 14. In this example, we assume that the exact solution of Equation (1) is

$$
u(x, y, t)=x^{2} y^{2} \sin (\pi x) \sin (\pi y) \sin (t), 0 \leq x, y, t \leq 1 .
$$

The values of initial and boundary conditions and $f$ can be achieved using the exact solution. The exact, Crank-Nicolson, and EDG solutions for $y=0.5, \operatorname{Re}=50, N=49$, and $\Delta t=0.01$ in different values of $t$ are shown in Figure 4(a) and in Figure 4(b), the absolute error of Crank-Nicolson method at $T=1$ for $\operatorname{Re}=50, N=49, \Delta t=0.01$, and $\alpha=0.5$ is depicted. Based on the Figures, we can see that the numerical solutions are a good approximation of the exact solutions. In Tables 3 and 4, the maximum of absolute errors and CPU Times for Crank-Nicolson and EDG methods for $\alpha=0.1,0.3$, and $\alpha=0.7,0.9$ with $T=1, \Delta t=0.01, \operatorname{Re}=100$, and different values of $N$ are tabulated, respectively. The EDG method generates the numerical solution with almost the same accuracy as the Crank-Nicolson method, but uses less time-consuming in comparison to the Crank-Nicolson method.

Example 15. Assume that the exact solution of Equation (1) is as follows:

$$
u(x, y, t)=t^{2} x^{2}(1-x)^{3} \sin (\pi y) \exp (x+y), 0 \leq x, y, t \leq 1 .
$$

The maximum of absolute errors and CPU Times for Crank-Nicolson and EDG methods for $\alpha=0.1,0.3,0.7,0.9$ with $T=1, \Delta t=0.01, \operatorname{Re}=5$, and different values of $N$ are shown in Tables 5 and 6, respectively. In Figure 5(a), the exact, Crank-Nicolson, and EDG solutions for $y=0.5, \mathrm{Re}$ $=20, N=49$, and $\Delta t=0.01$ in different values of $t$ are illustrated. Also, the absolute error of Crank-Nicolson method at $T=1$ for $\operatorname{Re}=20, N=49, \Delta t=0.01$, and $\alpha=0.5$ is portrayed in Figure 5(b). The numerical experiments verified our theoretical results once again.

Example 16. Consider Equation (1) with the exact solution

$$
u(x, y, t)=t^{2} \cos (\pi x) \cos (\pi y), 0 \leq x, y, t \leq 1 .
$$

This example does not apply to the initial and boundary conditions of the article, but the results of this example are as good as other examples.

In Tables 7 and 8, the maximum of absolute errors and the CPU time consumed by our proposed methods for $\alpha=$ $0.2,0.4$, and $0.6,0.8$ at $T=1, \Delta t=0.01, \mathrm{Re}=2$ with different mesh sizes are presented, respectively. Similar to other examples, the EDG method is faster than the Crank-Nicolson method. The exact, Crank-Nicolson, and EDG solutions for $y=0.5, \operatorname{Re}=10, N=49, \alpha=0.5$, and $\Delta t=0.01$ in different values of $t$ are displayed in Figure 6(a). In addition, the absolute error of the Crank-Nicolson method at $T=1, \alpha=0.5$, $\operatorname{Re}=10, N=49$, and $\Delta t=0.01$ is displayed in Figure 6(b).

Example 17. In this example, we assume that the exact solution of Equation (1) is

$$
u(x, y, t)=t^{2}\left(x-x^{2}\right)^{2}\left(y-y^{2}\right)^{2}, 0 \leq x, y, t \leq 1
$$

In Figure 7(a), the exact, Crank-Nicolson, and EDG solutions for $y=0.5, \operatorname{Re}=60, N=49$, and $\Delta t=0.01$ in different values of $t$ are shown. Besides, the absolute error of CrankNicolson is demonstrated in Figure 7(b). Obviously, our schemes are very accurate and quickly converge to the exact solution. The maximum of absolute errors and CPU Times for Crank-Nicolson and EDG methods for $\alpha=0.2,0.4$, and 
0.6, 0.8 at $T=1, \Delta t=0.01$, and $\operatorname{Re}=100$ with various mesh sizes are expressed in Tables 9 and 10, respectively.

\section{Conclusion}

In this paper, we introduced the Crank-Nicolson method and the EDG method which derived from $45^{\circ}$ rotation of the Crank-Nicolson approximation point and Taylor expansion to solve the 2D time-fractional Burgers' equation with Caputo-Fabrizio derivative. The error analysis and local truncation error of these methods gave in detail. The stability of the proposed numerical methods is analyzed by the Von-Neumann method. The convergence analysis of the $\mathrm{CN}$ and EDG methods proved. Some test problems chose to investigate the applicability and practical efficiency. From Tables 1-10, the results showed a good agreement with the exact solution, and the EDG method was faster than the $\mathrm{CN}$ method. Numerical experiments showed the efficiency of the proposed methods in terms of CPU time and accuracy.

\section{Data Availability}

All results have been obtained by conducting the numerical procedure and the ideas can be shared for the researchers.

\section{Conflicts of Interest}

The authors declare that there are no conflicts of interest regarding the publication of this paper.

\section{Acknowledgments}

We are very grateful to anonymous referees for their careful reading and valuable comments which led to the improvement of this paper.

\section{References}

[1] F. Abdullaev and V. V. Konotop, Nonlinear Waves: Classical and Quantum Aspects, Vol. 153, Springer Science \& Business Media, 2006.

[2] W. Gao, P. Veeresha, D. Prakasha, and H. M. Baskonus, "Novel dynamic structures of 2019-ncov with nonlocal operator via powerful computational technique," Biology, vol. 9, pp. 107-122, 2020.

[3] W. Gao, P. Veeresha, D. G. Prakasha, and H. M. Baskonus, "New numerical simulation for fractional benney-lin equation arising in falling film problems using two novel techniques," Numerical Methods for Partial Differential Equations, vol. 37, pp. 210-243, 2020.

[4] K. K. Ali, M. S. Osman, H. M. Baskonus, N. S. Elazabb, and E. İlhan, "Analytical and numerical study of the hiv-1 infection of $\mathrm{CD}^{+} \mathrm{T}$-cells conformable fractional mathematical model that causes acquired immunodeficiency syndrome with the effect of antiviral drug therapy," Mathematical Methods in the Applied Sciences, 2020.

[5] C. I. Muresan, P. Ostalczyk, and M. D. Ortigueira, "Fractional calculus applications in modeling and design of control systems," Journal of Applied Nonlinear Dynamics, vol. 6, pp. 131-134, 2017.
[6] C. Ionescu, A. Lopes, D. Copot, J. T. Machado, and J. Bates, "The role of fractional calculus in modeling biological phenomena: a review," Communications in Nonlinear Science and Numerical Simulation, vol. 51, pp. 141-159, 2017.

[7] H. Tang, D. Wang, R. Huang, X. Pei, and W. Chen, "A new rock creep model based on variable-order fractional derivatives and continuum damage mechanics," Bulletin of Engineering Geology and the Environment, vol. 77, pp. 375$383,2018$.

[8] Y. Zhuzhong, Z. Jiliu, and L. Fangnian, "Noise detection and image de-noising based on fractional calculus," Journal of Image and Graphics, vol. 19, pp. 1418-1429, 2014.

[9] X.-L. Gong, X.-H. Liu, and X. Xiong, "Chaotic analysis and adaptive synchronization for a class of fractional order financial system," Physica A: Statistical Mechanics and its Applications, vol. 522, pp. 33-42, 2019.

[10] M. Caputo and M. Fabrizio, "A new definition of fractional derivative without singular kernel," Progress in Fractional Differentiation and Applications, vol. 1, pp. 1-13, 2015.

[11] J. Shi and M. Chen, "A second-order accurate scheme for twodimensional space fractional diffusion equations with time caputo-fabrizio fractional derivative," Applied Numerical Mathematics, vol. 151, pp. 246-262, 2020.

[12] H. Qiao, Z. Liu, and A. Cheng, "A fast compact finite difference method for fractional Cattaneo equation based on CaputoFabrizio derivative," Mathematical Problems in Engineering, vol. 2020, Article ID 3842946, 17 pages, 2020.

[13] Z. Korpinar, "On numerical solutions for the Caputo-Fabrizio fractional heat-like equation," Thermal Science, vol. 22, pp. 8795, 2018.

[14] M. Zhang, Y. Liu, and H. Li, "High-order local discontinuous Galerkin method for a fractal mobile/immobile transport equation with the Caputo-Fabrizio fractional derivative," Numerical Methods for Partial Differential Equations, vol. 35, pp. 1588-1612, 2019.

[15] J. Singh, D. Kumar, Z. Hammouch, and A. Atangana, “A fractional epidemiological model for computer viruses pertaining to a new fractional derivative," Applied Mathematics and Computation, vol. 316, pp. 504-515, 2018.

[16] Z. Liu and X. Li, "A Crank-Nicolson difference scheme for the time variable fractional mobile-immobile advection-dispersion equation," Journal of Applied Mathematics and Computing, vol. 56, pp. 391-410, 2018.

[17] R. H. Shariffudin and A. R. Abdullah, "Hamiltonian circuited simulations of elliptic partial differential equations using a spark," Applied Mathematics Letters, vol. 14, pp. 413-418, 2001.

[18] N. Abdi, H. Aminikhah, A. H. Refahi Sheikhani, and J. Alavi, "A high-order compact alternating direction implicit method for solving the 3D time-fractional diffusion equation with the Caputo-Fabrizio operator," Mathematical Sciences, vol. 14, pp. 359-373, 2020.

[19] J. Jia and H. Wang, "A fast finite volume method on locally refined meshes for fractional diffusion equations," East Asian Journal on Applied Mathematics, vol. 9, pp. 755-779, 2019.

[20] H. Chen, S. Lü, and W. Chen, "Spectral methods for the time fractional diffusion-wave equation in a semi-infinite channel," Computers \& Mathematics with Applications, vol. 71, pp. 1818-1830, 2016.

[21] Z. Chen, G. Huan, and Y. Ma, Computational Methods for Multiphase Flows in Porous Media, SIAM, 2006. 
[22] A. R. Abdullah, "The four point explicit decoupled group (EDG) method: a fast poisson solver," International Journal of Computer Mathematics, vol. 38, pp. 61-70, 1991.

[23] M. K. M. Akhir, M. Othman, Z. A. Majid, M. Suleiman, and J. Sulaiman, "Four point explicit decoupled group iterative method applied to two-dimensional Helmholtz equation," International Journal of Mathematical Analysis, vol. 6, pp. 963-974, 2012.

[24] A. M. Saeed, "Solving 2d time-fractional diffusion equations by preconditioned fractional edg method," Progressive Research in Mathematics, vol. 14, pp. 1-7, 2018.

[25] A. T. Balasim and N. H. M. Ali, "Group iterative methods for the solution of two-dimensional time-fractional diffusion equation," in AIP Conference Proceedings 1750, 030003, 2016.

[26] J. Eng, A. Saudi, and J. Sulaiman, "Performance analysis of the explicit decoupled group iteration via five-point rotated Laplacian operator in solving poisson image blending problem," Indian Journal of Science and Technology, vol. 11, no. 12, pp. 1-6, 2018.

[27] D. Kong, Y. Xu, and Z. Zheng, "A hybrid numerical method for the KdV equation by finite difference and sinc collocation method," Applied Mathematics and Computation, vol. 355, pp. 61-72, 2019.

[28] Z. Liu, A. Cheng, and X. Li, “A second order Crank-Nicolson scheme for fractional Cattaneo equation based on new fractional derivative," Applied Mathematics and Computation, vol. 311, pp. 361-374, 2017.

[29] J. M. Holte, Discrete Gronwall Lemma and Applications, vol. 24, MAA-NCS meeting at the University of North Dakota, 2009. 